\title{
The Centaurus A Northern Middle Lobe as a Buoyant Bubble
}

\author{
Curtis J. Saxton ${ }^{1,2}$ \\ and \\ Ralph S. Sutherland ${ }^{2}$ \\ and \\ Geoffrey V. Bicknell ${ }^{2,1}$
}

\begin{abstract}
We model the northern middle radio lobe of Centaurus A (NGC 5128) as a buoyant bubble of plasma deposited by an intermittently active jet. The extent of the rise of the bubble and its morphology imply that the ratio of its density to that of the surrounding ISM is less than $10^{-2}$, consistent with our knowledge of extragalactic jets and minimal entrainment into the precursor radio lobe. Using the morphology of the lobe to date the beginning of its rise through the atmosphere of Centaurus A, we conclude that the bubble has been rising for approximately $140 \mathrm{Myr}$. This time scale is consistent with that proposed by Quillen et al. (1993) for the settling of post-merger gas into the presently observed large scale disk in NGC 5128, suggesting a strong connection between the delayed re-establishment of radio emission and the merger of NGC 5128 with a small gas-rich galaxy. This suggests a connection, for radio galaxies in general, between mergers and the delayed onset of radio emission. In our model, the elongated X-ray emission region discovered by Feigelson et al. (1981), part of which coincides with the northern middle lobe, is thermal gas that originates from the ISM below the bubble and that has been uplifted and compressed. The "large-scale jet" appearing in the radio images of Morganti et al. (1999) may be the result of the same pressure gradients that cause the uplift of the thermal gas, acting on much lighter plasma, or may represent a jet that did not turn off completely when the northern middle lobe started to buoyantly rise. We propose that the adjacent emission line knots (the "outer filaments") and star-forming
\end{abstract}

\footnotetext{
${ }^{1}$ Department of Physics \& Theoretical Physics, Faculty of Science, Australian National University, ACT 0200, Australia

${ }^{2}$ Research School of Astronomy \& Astrophysics, Mt Stromlo Observatory, Australian National University, Cotter Road, Weston ACT 2611, Australia
} 
regions result from the disturbance, in particular the thermal trunk, caused by the bubble moving through the extended atmosphere of NGC 5128.

Subject headings: hydrodynamics - ISM: bubbles — galaxies: active galaxies: individual (Centaurus A, NGC 5128) — galaxies: jets — radio continuum: galaxies

\section{INTRODUCTION}

Centaurus A, as the closest active galaxy that is also radio loud has always attracted a lot of attention with numerous papers covering the electromagnetic spectrum from radio through to gamma-rays. Its proximity means that various physical processes can be studied in detail with high spatial resolution and from time to time new observational data appear that confront our understanding of this object and the physical processes occurring in radio galaxies generally. The recent observations of the northern middle lobe (NML) of Centaurus A by Morganti et al. (1999) and the related observations of star formation and emission-line clouds in its vicinity (Graham \& Price 1981; Morganti et al. 1991; Graham 1998; Sutherland et al. 1993; Mould et al. 2000) fall into this category. The radio observations of the NML are the first published observations of the NML and fill in the spatial gap between the extant VLA images of the inner lobe and the large scale structure. The curious structure of the NML and its associated "large-scale jet" linking it the inner lobe beg explanation as does the apparent association with the star-forming and emission-line regions. We propose such an explanation in this paper and in so doing link the formation of multi-lobed structure in Centaurus A to its aspherical X-ray morphology and its merger history. Our work is based in the growing recognition of the importance of buoyant effects in the structure of radio galaxies. Churazov et al. (2001) have presented a convincing model of the M87 radio structures and associated X-ray features proposing that the intermediate radio structures in that galaxy (Owen et al. 2000) can be modeled in terms of radio lobes rising buoyantly in the gravitational potential. In this paper we propose a similar model for Centaurus A albeit with some differences that are related to different aspects of Centaurus A. In particular, we concentrate on the timescale and history of the bubble's rise, and its possible relationship with the merger history of NGC 5128 (Tubbs 1980; Bland et al. 1987; Nicholson et al. 1992; Quillen et al. 1993; Sparke 1996).

In section 2 we provide an overview of observations of Centaurus A and relevant theoretical studies of buoyant bubbles in extragalactic contexts. Section 3 describes the method of our simulations. Details of the numerical results and the implications for the physical system are described in $\S 4$. A discussion of the main features of this work is given in $\S 5$. 


\section{SUMMARY OF RELEVANT OBSERVATIONS \& THEORY}

\subsection{Previous observations}

\subsubsection{Galaxy, atmosphere, shells, \& HI clouds}

The giant elliptical galaxy Centaurus A (NGC 5128) is the closest large radio galaxy, at a distance of $3.6 \pm 0.2 \mathrm{Mpc}$ (Soria et al. 1996) where $1^{\prime}$ corresponds to a projected distance of $1.0 \mathrm{kpc}$. A warped dust and gas disk through the middle of the galaxy (see e.g. Tubbs 1980; Bland et al. 1987; Nicholson et al. 1992; Quillen et al. 1992; Quillen et al. 1993) is probably a relic of a disk galaxy consumed in a merger. Stellar shells (Malin et al. 1983) and HI clouds are distributed out to distances of several tens of kpc from the nucleus. They may also be merger relics. The cloud locations may be associated with some of the stellar shells (Schiminovich et al. 1994). Richter et al. (1994) found the mass of HI clouds around Centaurus A to be $7.8 \times 10^{8} \times(1.0 \pm 0.3) M_{\odot}$. Schiminovich et al. (1994) found an HI mass of $4.5 \times 10^{8} M_{\odot}$ in the disk, and $1.5 \times 10^{8} M_{\odot}$ in the shells, peaking at a projected distance $15 \mathrm{kpc}$ from the nucleus.

The galaxy has an atmosphere of X-ray emitting gas (Feigelson et al. 1981). Turner et al. (1997) observed diffuse X-ray emission within $6^{\prime}$ of the nucleus, and concluded that there are two thermal components, with $k T=0.29 \mathrm{keV}$ and $5 \mathrm{keV}$. They suggest that the hotter component may be related to binary stellar systems, but the possibility that it is thermal gas cannot be excluded (e.g. Wagner et al. 1996). If interpreted as thermal gas, the sound speeds corresponding to these temperatures are $2.7 \times 10^{2} \mathrm{~km} \mathrm{~s}^{-1}$ and $1.1 \times 10^{3} \mathrm{~km} \mathrm{~s}^{-1}$. The stellar velocity dispersion ranges from $\sigma_{*} \approx 143 \mathrm{~km} \mathrm{~s}^{-1}$ to $90 \mathrm{~km} \mathrm{~s}^{-1}$ at locations $2.8 \mathrm{kpc}$ to $20 \mathrm{kpc}$ from the nucleus (Hui et al. 1995). This corresponds to a parameter $\beta \equiv T_{*} / T=$ $\mu m_{p} \sigma_{*}^{2} / k T=0.46$ for gas at a temperature of $0.29 \mathrm{keV}$ but $\beta=0.03$ if $k T=5 \mathrm{keV}$. The former is in better agreement with the X-ray profile of Forman et al. (1985); it suggests that the stellar velocity dispersion is a modest fraction of the virial value.

Marconi et al. (2000) observed the nuclear region in the near-infrared, where obscuration by the dusty disk is minimal, and determined the radial profile for the stellar distribution near the core. Fitting these observations to the empirical "Nuker law" model of Lauer et al. (1995), yielded a core radius of $3^{\prime \prime} .3-5^{\prime \prime} .6$ with a best fit of $3^{\prime \prime} .9$, corresponding to $r_{0}=78 \mathrm{pc}$. This should be approximately the value of the core radius of the entire galaxy, since the stellar component dominates near the nucleus.

The mass of Cen A was estimated from measurement of the X-ray halo, to be $6 \times 10^{11} M_{\odot}$ within $<10 \mathrm{kpc}$ by Forman et al. (1985) who assumed a temperature $k T=1.0 \mathrm{keV}$. This should be revised to $1 \times 10^{11} M_{\odot}$ using the precise temperature of $k T=0.29 \mathrm{keV}$ measured 
by Turner et al. (1997). Such a value is consistent with the estimate of $1.8 \times 10^{11} M_{\odot}$ within the span of the disk, and the estimate of $2 \times 10^{11} M_{\odot}$ within $15 \mathrm{kpc}$ by Schiminovich et al. (1994) from velocity measurements of the HI shells, assuming circular motion. Kinematic studies of the planetary nebulae (Hui et al. 1995; Mathieu et al. 1996) indicate a mass of $3.1 \times 10^{11} M_{\odot}$ within $25 \mathrm{kpc}$ and $(3-5) \times 10^{11} M_{\odot}$ within $50 \mathrm{kpc}$.

\subsubsection{Radio features}

Cen A possesses radio-emitting features on diverse scales. A radio jet extends a projected distance $\sim 6 \mathrm{kpc}$ from the nucleus (e.g. Jones et al. 1996), and is visible out to $\sim 4 \mathrm{kpc}$ in X-rays (e.g. Turner et al. 1997). Radio plasma from the jets accumulates in inner radio lobes which extend to projected distances $\sim 8 \mathrm{kpc}$ from the nucleus, peak at $\sim 6 \mathrm{kpc}$ and are $\sim 4 \mathrm{kpc}$ across at the widest points. Vast regions of fainter emission extend beyond the inner lobes. In projection, the outer lobes comprise the largest object on the sky, extending across $10^{\circ}$ (Cooper et al. 1965).

Morganti et al. (1999) studied the radio emission emanating from the northern middle lobe and its immediate surroundings. The NML structure extends to at least $40 \mathrm{kpc}$ from the nucleus, and the brightness peaks at $23 \mathrm{kpc}$ in projection (see Figure 1 for a grey-scale representation of their Figure 2 contour image).

Near the brightest part of the NML Feigelson et al. (1981) detected diffuse soft X-ray emission from a region extending $1 \mathrm{kpc}$ wide and a projected height of $5 \mathrm{kpc}$ in the direction away from the galaxy. By their interpretation, if the X-ray emitting region is composed of thermal gas at temperatures $\sim 10^{6} \mathrm{~K}$, compressed from the surrounding medium then it must be ephemeral, with timescales of $\sim 10 \mathrm{Myr}$ and $\sim 1 \mathrm{Myr}$ for cooling and dynamical expansion respectively. They also considered an alternative synchrotron model with an electron population $10^{2}$ Myr old. Morganti et al. (1999) found a $\sim 2^{\prime}$ wide radio-emitting structure connecting the NML to the inner lobe. They named this the "large-scale jet" and noted that its direction is aligned within a few degrees of the projected extension of the nuclear jet.

\subsubsection{Optical filaments and star-forming regions}

Blanco et al. (1975) discovered several filamentary ionized knots in the vicinity of the NML. Figure 7 of Morganti et al. (1999) shows the positions of the filaments in relation to the radio emitting structures and an HI cloud at the western margin of the NML and 
"large-scale jet." In projection, the inner filaments start at $7.8 \mathrm{kpc}$ from the nucleus (i.e. close to the top of the northern lobe) and extends a further $2 \mathrm{kpc}$, in a direction parallel to the "large-scale jet". Similarly, outer filaments exist at $13.6 \mathrm{kpc}$ in projection, extending a further $8 \mathrm{kpc}$ and are coincident with the southern edge of the NML. The outer filaments appear projected at the southern edge of the NML, and on the eastern edge of a $6 \times 10^{6} M_{\odot}$ HI cloud (Morganti et al. 1999). Proposed mecahnisms for the excitation of the filaments include beamed emission from the nucleus (Morganti et al. 1991) or autoionizing shocks from local disturbances associated with the radio plasma (Sutherland et al. 1993). The inner filaments show a spread of velocities $\sim 360 \mathrm{~km} \mathrm{~s}^{-1}$, up to $\sim 430 \mathrm{~km} \mathrm{~s}^{-1}$ blue-shifted relative to the galaxy (Morganti et al. 1991), whereas the components of the outer filaments are blue-shifted by $\lesssim 300 \mathrm{~km} \mathrm{~s}^{-1}$, with two outliers blue-shifted by $\sim 500 \mathrm{~km} \mathrm{~s}^{-1}$, (Graham 1998).

Graham (1998) observed a concentration of young stars between the outer filaments and the HI cloud near the southwest edge of the NML. Mould et al. (2000) determined that the stars are of age $\approx 16 \mathrm{Myr}$.

Both Graham (1998) and Mould et al. (2000) suggest that the star-formation is shockinduced by disturbances associated with the NML and/or the "large-scale jet". The HI cloud has sufficient velocity for it to have traversed the disturbed region by a distance equivalent to its own width during the age of the stars; it is most likely the source of star-forming gas.

\subsection{Previous simulations of buoyant bubbles}

Churazov et al. (2001) performed hydrodynamic simulations of a rising buoyant bubble in order to explain the radio and X-ray morphology of M87 out to a distance of $50 \mathrm{kpc}$. They used an Eulerian ZEUS-3D code. Magnetic fields were assumed to be tangled on small scales, with a local proportionality between $P$ and $B^{2}$. The initial bubble of radio plasma was placed $9 \mathrm{kpc}$ from the nucleus, with a radius of $5 \mathrm{kpc}$. Its density contrast relative to ambient gas was $\eta=10^{-2}$. The undisturbed galaxy atmosphere profile was that of Nulsen \& Böhringer (1995). The $200 \times 2002 \mathrm{D}$ grid extended to a radius of $51 \mathrm{kpc}$, in spherical coordinates. The energy distribution of the radio-emitting relativistic plasma was determined by using 2000 tracer particles to follow the energy loss history within the bubble, assuming local pressure proportionality between magnetic field and thermal pressure.

Churazov et al. (2001) found that: (1) in the absence of surface tension an initially

spherical bubble deforms into a torus as it rises, on timescales of $\sim 10 \mathrm{Myr}$; (2) thermal gas is drawn up from below the bubble, and is overdense compared to the ambient gas; (3) 
the bubble reaches a maximum height at which its density equals that of the surrounding medium; (4) thereupon the bubble spreads laterally, forming a "pancake"; (5) convection of the atmosphere surrounding the bubble flattens the local gradients of density and X-ray emissivity. The shapes of the simulated mushroom-cloud radio bubble and thermal gas trunk are qualitatively consistent with the radio and X-ray features surrounding M87. (I.e. regions of diffuse X-ray excess just radially inwards from the radio lobes; these correspond to thermal gas trunks trailing bubble tori.)

Brüggen \& Kaiser (2001) studied a bubble rising through the medium of a galaxy cluster, using the same method and resolution as Churazov et al. (2001) but with different scales, geometric proportions and ambient atmosphere model. The initial bubble size and location were on the order of a core radius of the cluster atmosphere. Results were qualitatively consistent with Churazov et al. (2001), except that a stalk of radio plasma was left on the axis behind the ascending bubble. Elongation of the initial bubble promoted RaleighTaylor instabilities which altered the evolution. Fluid instabilities that ordinarily distort the bubble shape were inhibited in simulations including a strong $\left(B^{2} \sim P\right)$ magnetic field that was initially parallel to the bubble surface.

\section{SIMULATION OF THE CENTAURUS A NORTHERN MIDDLE LOBE}

\subsection{Method and initial conditions}

Our simulations were performed using a piecewise parabolic method, PPM, (Colella \& Woodward 1984). The code, PPMLR, is adapted from the VH-1 hydrocode (http://wonka.physics.ncsu . with efficiency improved by Sutherland et al. (2001). It is an explicitly conservative secondorder Gudonov scheme with Lagrangian advection followed by remapping to a fixed Eulerian grid.

Our basic calculations were carried out on an axially symmetric grid with a resolution $600 \times 300$ in the $z$ and $R$ directions respectively. The physical span of the grid was $1000 r_{0} \times$ $500 r_{0}$. To test the robustness of results, supplementary calculations were performed at half the basic resolution. Further simulations were done at triple the standard resolution but with a third the standard duration to investigate some fine morphological details. Initially the gas density and pressure were distributed according to an isothermal $\beta$-model with the number density, $n$ described by:

$$
n=n_{0}\left[1+\frac{r^{2}}{r_{0}^{2}}\right]^{-\beta}
$$

Note that the so-called $\beta$-model widely used in the literature is wrong (see Bicknell (1995); 
Killeen \& Bicknell (1988) for a discussion) so that the value of $\beta=0.75$ used by us is equal to the Forman et al. $(1985)$ value $(\approx 0.5 \pm 0.1)$ times $3 / 2$. The value of $\beta$ implied by the stellar and gas temperatures is $\beta_{g}=\mu m_{p} \sigma_{*}^{2} / k T \approx 0.46$ for $\sigma=143 \mathrm{~km} \mathrm{~s}^{-1}$ (at approximately 36 core radii) and $k T \approx 0.29 \mathrm{keV}$. (The stellar population may be at a temperature about $\sim 0.6$ times the virial temperature.)

The nucleus of the galaxy is the coordinate origin, positioned halfway along the grid's $z$-axis. Following equation (3-1), above, the adopted density and pressure profiles of the interstellar medium (ISM) are:

$$
\begin{aligned}
& \rho=\rho_{0}\left(1+\xi^{2}\right)^{-\beta}, \\
& P=P_{0}\left(1+\xi^{2}\right)^{-\beta},
\end{aligned}
$$

where

$$
\xi \equiv \frac{r}{r_{0}}
$$

is the scaled distance from the nucleus, and $\rho_{0}, P_{0}$ are density and pressure values at the nucleus.

We establish initial conditions such that there is initially no flow. The gravitational and pressure forces balance everywhere. The gravitational field is thereby inferred from the $\beta$-model pressure gradient. That is, the gravitational force per unit mass implied by this model is

$$
f=\frac{1}{\rho} \frac{d P}{d r}=\frac{-2 \beta \xi}{1+\xi^{2}} \frac{P_{0}}{\rho_{0} r_{0}} .
$$

The bubble is initially located on the $z$-axis at $z_{b}=60 r_{0}$ with a spherical radius $r_{b}=$ $20 r_{0}$. The ratio of the bubble's radius to its height, $r_{b} / z_{b}$ is approximately the same as the projected proportions of the northern inner lobe of Cen A, $r / z \approx 2 \mathrm{kpc} / 6 \mathrm{kpc}$. The pressure and density of the $\beta$-model at the level $\xi_{b}=z_{b} / r_{0}$ are used to set the constant pressure and density everywhere within the bubble. The bubble pressure and density are prescribed by

$$
\begin{aligned}
P & =P_{0}\left(1+\xi_{b}^{2}\right)^{-\beta} \text { and } \\
\rho & =\eta \rho_{0}\left(1+\xi_{b}^{2}\right)^{-\beta},
\end{aligned}
$$

where $\eta$ is the ratio of the bubble density to the ambient density at $z=z_{b}$. We have investigated cases of bubbles with $\eta=0.5,10^{-2}$ and $10^{-3}$. Figure 2 shows an initial density image of the galaxy atmosphere and the bubble.

In order to track the different gases (i.e. radio emitting plasma and the ISM), we have implemented a scalar tracer variable $\varphi$, which is passively advected with the fluid. The tracer variable, $\varphi$, is constant along a stream line so that

$$
\frac{d \varphi}{d t} \equiv \frac{\partial \varphi}{\partial t}+v_{\alpha} \frac{\partial \varphi}{\partial x_{\alpha}}=0 .
$$


This is implemented in the code in the conservative form:

$$
\frac{\partial}{\partial t}(\rho \varphi)+\frac{\partial}{\partial x_{\alpha}}\left(\rho \varphi v_{\alpha}\right)=0 .
$$

Bubble material initially has a tracer value $\varphi=1$, and non-bubble gas has $\varphi=0$. In each cell of the evolving simulation, the value of $\varphi$ therefore indicates the volumetric fraction of material originating within the initial bubble, i.e. $\varphi$ traces the local distribution of radio plasma as distinct from thermal gas.

\subsection{Scaling}

The gas dynamic equations are scaled according to the following prescription. First, the unit of length is taken as the core radius of the ISM profile, $r_{0}$. However, it is not crucial that we match this exactly with the observed core radius of the galaxy for the following reason: The bubble is located well outside a core radius where the number density and pressure follow a power law in $r / r_{0}$. Hence, $r_{0}$ is in reality a scaling radius and as we show below (§4.1.4), it is necessary to adopt a value of $r_{0}$ approximately twice the optical core radius in order to match the simulations and the observed physical scale of the NML.

Second, the velocity scale is the isothermal sound speed of the ISM,

$$
v_{0}=\sqrt{\frac{k T}{\mu m_{p}}}=210 \mathrm{~km} \mathrm{~s}^{-1}\left(\frac{k T}{0.29 \mathrm{keV}}\right) .
$$

Third, the time scale is

$$
t_{0} \equiv \frac{r_{0}}{v_{0}}=0.25 \mathrm{Myr}\left(\frac{r_{0}}{100 \mathrm{pc}}\right)\left(\frac{k T}{\mathrm{keV}}\right)^{-1 / 2},
$$

\section{RESULTS}

\subsection{Evolution}

\subsubsection{Physical components \& velocity field}

Let us now describe the general characteristics of our simulations. This description is aided by the images of density and velocity vector fields in Figures 2-6; and the plots in Figures 7-10. 
Bubble plasma rises buoyantly in the ambient gas (density $\rho_{\mathrm{a}}$ ) with acceleration $\sim$ $\left(1-\rho_{\mathrm{b}} / \rho_{\mathrm{a}}\right) f(f=$ local gravitational acceleration $)$. The thermal gas circulates around the bubble and fills the space behind and beneath the bubble.

As decribed by Churazov et al. (2001), in the early stages of the simulation a trunk of dense thermal gas moves up along the $z$-axis from regions immediately beneath the bubble. This uplift is driven by a rarefaction that develops behind the rising bubble and which persists, albeit weaker, as the bubble develops into a torus. It penetrates the bubble from below, and makes contact with the thermal gas on the upper side, (e.g. Figures 3, 4), thereby turning the bubble from a sphere to the shape of a mushroom-cap and then into a torus. When the trunk breaks through the top of the bubble (e.g. Figure 3), velocities at the interface between the uplifted thermal gas and the bubble can temporarily exceed the sound speed of the ambient gas, as indicated by the early peak in $\left|v_{\max }\right|$ in each of the curves of maximum velocity against time in Figure 7.

The torus rises buoyantly, and the majority of the radio plasma remains within it throughout our simulations ( to $t=1200 t_{0}$ ) although there is some peripheral mixing between the radio plasma and the ambient gas. At some stages the primary torus has a circular cross-section (e.g. Figure 5), but at other times the cross-section is highly flattened (e.g. Figure 6).

As it rises, the torus intermittently sheds minor vortex rings. These rings occur closer to the galaxy, have lower density contrasts relative to their surroundings, and typically propagate down towards the nucleus of the galaxy.

Thermal gas circulates around the main bubble torus and also around many of the blobs of plasma that separate from the main bubble. The disturbed gas is drawn towards the $z$-axis on the underside of the blob, vertically upwards on the inner radial side, and radially outwards across the upper surface. The velocity of the vortex ring increases towards the nearest torus surface, but drops to zero in the central eye of the circulation (see e.g. the prominent vortex rings in Figure 5$)$. At late stages $\left(t \gtrsim 200 t_{0}\right)$ the maximum velocities are typically $\sim 0.7 v_{0}$ (Figure 7 ), which is a large fraction of the adiabatic sound speed, $\sqrt{5 / 3} v_{0}$. Greater maximum velocities occur at earlier times in the cases of $\eta=10^{-2}, 10^{-3}$; the maximum velocities are typically $\approx 1.2 v_{0}$ until $t \approx 300 t_{0}$ (e.g. the system in Figure 3 where the thermal gas trunk has just broken through the bubble).

The early-stage thermal gas trunk persists as a local region of overdense, rapidly uplifted gas immediately beneath and bounded by the major torus. At times $t \sim 120 t_{0}-150 t_{0}$, soon after the trunk penetrates the top of the mushroom, the most overdense area of the trunk is typically twice as dense as the $\beta$-model density at the same $\xi$ coordinate. In the $\beta=0.75$ 
model this is equivalent to the undisturbed density contour at a location $\approx 0.6$ times as close to the nucleus. (See the clear overdensity of thermal gas in regions encompassed by the torus in, for example, Figures 4,5.)

At sufficiently late stages $\left(t \gtrsim 150 t_{0}\right)$ the torus is high and wide enough that its uplifting influence on the lower parts of the trunk is weaker. Beneath the uplift region there is a stagnant region, and further beneath this the unsupported gas slumps towards the galaxy under gravity. The slump is transonic with approximately the same maximum velocity as the uplift region. The slumping region is $\gtrsim 40 r_{0}$ away from the major torus. (See Figure 4 where a weak, low-velocity slump is commencing close to the galaxy, and Figures 5,6 in which the slump is fully developed and transonic throughout much of the thermal gas trunk.)

\subsubsection{Ascent of the radio plasma to the neutral buoyancy point}

The evolving bubbles rise until reaching a neutral buoyancy point, where the bubble density equals the ambient gas density. By equating the undisturbed ISM density (3-2) with the initial bubble density (3-7), a theoretical upper altitude is estimated, $\xi_{\text {final }}=$ $\left[\left(1+\xi_{b}^{2}\right) \eta^{-1 / \beta}-1\right]^{1 / 2} \sim \xi_{b} \eta^{-1 / 2 \beta}$. When $\xi_{b}=60$ and $\beta=0.75$, the limit is $\xi_{\text {final }} \approx$ $95,1.3 \times 10^{3}$ and $6.0 \times 10^{3}$ for $\eta=0.5,0.01$ and 0.001 respectively. In practice, these values are upper limits because mixing and entrainment of thermal gas may increase the effective value of $\eta$ as radio plasma rises.

Figure 8 shows the inner $\left(\xi_{\min }\right)$ and outer $\left(\xi_{\max }\right)$ radial extremes of the bubble plasma as determined by the distribution of the tracer $\varphi$, for $\beta=0.75$ and $\eta=0.5,10^{-2}, 10^{-3}$. In the case $\eta=0.5$ the bubble achieves neutral buoyancy when $t \approx 400 t_{0}$. At this stage the median bubble position agrees with the prediction for material originating in the middle of the initial bubble, $\left(\xi_{\min }+\xi_{\max }\right) / 2 \approx 80 \lesssim \xi_{\text {final }}$.

The cases $\eta=10^{-2}, 10^{-3}$ are not clearly separated in their $\xi_{\min }$ and $\xi_{\max }$ evolutions because both the respective $\xi_{\text {final }}$ values are far beyond the limits of our numerical grid. However the lateral spreading of bubble material (Figure 9) is different in the $\eta=10^{-2}, 10^{-3}$ cases: for $\eta=10^{-3}$ the radio plasma is more widely distributed perpendicular to the $z$-axis after $t \approx 500 t_{0}$ (compare the upper two curves of Figure 9 ). In general the rate of lateral spreading declines after $t \sim 600 t_{0}$ (lower curve of Figure 9), and the spreading is greater for cases with lower $\eta$. 


\subsubsection{Effect of $\beta$}

Figure 10 shows the time-evolution of $\xi_{\min }, \xi_{\max }$ for different galaxy $\beta$-models, with $\eta=10^{-2}$. The outer front, $\xi_{\max }$, is not very sensitive to $\beta$; however the inner radius $\xi_{\min }$ and morphology of bubble plasma are affected by $\beta$. In early phase of the bubble evolution $\xi_{\text {min }}$ increases with time as the bubble rises and is penetrated by the thermal trunk. For $\beta=0.75,0.9$ a droplet of radio plasma remains on the axis in the middle of the thermal trunk, and it sinks slowly until the time $t \approx 250 t_{0}$ when it is too dispersed to be resolved numerically in $\varphi$ data. No such droplet appears in results for lower $\beta$ values.

At some medium time $\left(t>200 t_{0}\right)$ in the evolution of each bubble, the torus has widened and a wisp of radio plasma is far enough from the uplift region to become caught in the slump of thermal gas on the $z$-axis. This wisp becomes a rapidly descending radio stalk, with $r_{\min }$ decreasing in time at a rate of $\approx 0.5 v_{0}$. The time of stalk formation is sensitive to $\beta$ : it occurs at $t \approx 210 t_{0}, 250 t_{0}, 400 t_{0}, 600 t_{0}$ for cases of $\beta=0.9,0.75,0.5,0.375$ respectively and $\eta=10^{-2}$. The stalk descends to a distance from the core approximately equivalent to the lower surface of the initial bubble.

\subsubsection{Rise and scaling of the bubble}

We can compare the distribution of bubble material in simulations with the distribution of the NML and northern inner lobe, in order to limit and deduce the plausible ranges of system parameters and the age of the NML. With a scale of $r_{0}=0.10 \mathrm{kpc}$, none of our simulations ends with a substantial fraction of the bubble material at or above the height of the peak radio brightness of the NML, i.e. $23 \mathrm{kpc}$ from the nucleus in projection (Figures 8,10 ). The bubble with $\eta=0.5$ rises by less than its own initial diameter; therefore the NML must have a smaller value of $\eta$. With $\eta=10^{-2}, 10^{-3}$ a factor $\sim 3$ rise in the top of the bubble matter is possible by a time $\sim 10^{3} t_{0}$, and a factor $\sim 2$ is possible by the time $200 t_{0}$.

In order to match the simulated and observed radio morphologies (as shown below in $\S 4.2 .3$ ), and to have circulation velocities high enough to explain the velocity spread of the outer filaments (recall §4.1.1), we propose an age of $<200 t_{0}$. For a simulated bubble of this age to be at a height corresponding to the NML, we must rescale the linear unit of the simulation to $r_{0} \approx 0.2 \mathrm{kpc}$. This implies that the NML precursor must have been roughly twice as big and far from the galaxy as the present inner lobe. Such rescaling is valid because at all stages of the bubble's evolution it is in the power-law region of the ISM structure, far from the core (§3.2). The correspondingly revised time unit is $t_{0} \approx 0.92 \mathrm{Myr}$. 


\subsection{Simulated radio morphology}

\subsubsection{Method and morphological identification}

Assuming that the magnetic field is tangled on small scales and that its pressure is proportional to the gas pressure, the synchrotron volume emissivity per unit frequency is $j_{\nu} \propto \varphi P^{(3+\alpha) / 2}$, where $\alpha \approx 0.6$ is the spectral index. Surface brightness maps are rendered by revolving the axially symmetric pressure and tracer data into three-dimensional cylindrical structures, and then numerically integrating $j_{\nu}$ along the line of sight for each pixel of the surface brightness image.

We define characteristic stages of the simulated evolving radio morphology, as depicted in high resolution in Figure 11, and with comparisons in $\eta$ in Figure 12. these are compared with observational radio images (Morganti et al. 1999) to indicate approximately how long ago the NML began its buoyant rise. The evolutionary stages we define can be described as:

- "initial bubble": limb-darkened circle; $t=0$ (e.g. see top-left panel of Figure 11);

- "mushroom cap": limb-darkened mushroom shape, dimpled at the bottom, as the thermal gas trunk penetrates the side closest to the galaxy and sweeps radio plasma upwards on the $z$-axis; the edge furthest from the galaxy points in the positive $z$ direction with fainter emission; $t \lesssim 80 t_{0}$ (e.g. see $t=60 t_{0}$ panel of Figure 11 );

- "young torus": thermal trunk has completely penetrated the bubble, deforming it into a torus; bright torus is narrow compared with the central hole; a wispy 'hat' of emission extends above the main torus; $80 t_{0} \lesssim t \lesssim 200 t_{0}$ (e.g. see $t=140 t_{0}$ panel of Figure 11 );

- "torus + stalk": wider bright torus; some faint skirts close to the torus, above and mainly below; with wispy emission from a stalk-like feature descending on the $z$-axis; $t \sim 10^{2.5} t_{0}$ (e.g. see $t=380 t_{0}$ panel of Figure 11 );

- "old torus + stalk + rings": wider, higher torus, with a stalk and numerous wispy rings of emission partially filling the space between the main torus and the galaxy; highest extent of bubble material is $\approx 3$ times further from galaxy than in the initial condition; $t \sim 10^{3} t_{0}$ (e.g. see the $t=1200 t_{0}$ panels of Figure 12)

\subsubsection{Appearance of the torus and inclination}

At stages with a well-defined torus, the apparent morphology depends on orientation. When the $z$-axis is at a small or medium angle to the line of sight (inclination $\lesssim 70^{\circ}$ ) the 
torus appears as a bright ring with an aspect ratio depending on inclination. Thus Churazov et al. (2001) identify rings observed in radio images of M87 with buoyantly rising tori (e.g. in their Figure 1) viewed at an angle $\sim 45^{\circ}$. When the torus is viewed almost edge-on (inclination $\gtrsim 70^{\circ}$ ) it appears as a bright bar transverse to the $z$-axis, with darkened edges and a somewhat darkened middle (see Figure 13). The brightest part of the NML of Cen A appears as a transverse bar in the radio images of Morganti et al. (1999) (see Figure 1). If, as our model suggests, this is a buoyant torus, then its apparent aspect ratio implies an inclination $\approx 73^{\circ}$. This estimated inclination is within the observational limits of Tingay et al. (1998) for the direction of the radio jet on parsec scales. Furthermore, if the NML is at a toroidal stage, its age has a lower limit, $t \gtrsim 80 t_{0}$.

\subsubsection{Fainter radio features}

Radio-emitting wisps and skirts occur when radio plasma is sheared from the torus and entrained into the thermal gas. Wisps complicate the morphology increasingly in later stages. Firstly, as the mushroom cap is deformed into a "young torus," some radio plasma displaced by the upthrusting thermal trunk is redistributed above the torus, resembling a "fuzzy hat" of faint emission above the bar (see e.g. the case of $t=150 t_{0}, \eta=10^{-3}$ in Figure 12). As gas circulates around the widening torus, wisps are drawn outwards across the top of the torus, downwards across the outside, and inwards towards the $z$-axis along the underside of the torus. At some times the wisps are evenly distributed above and below the torus (see e.g. the cases of $t=300 t_{0}$ in Figure 12). Ultimately, in the "old torus" stage, the space between the torus and the galaxy becomes crowded with radio-emitting vortex rings and skirts that descended after being sheared off the torus (see e.g. the cases of $t=1200 t_{0}$ in Figure 12).

In detail the occurrence of minor radio-emitting wisps is sensitive to $\eta$, the initial geometry and ambient gas/potential profile. For instance the cases of $\eta=10^{-2}$ and $\eta=10^{-3}$ but the same initial geometry show different particular distributions of wispy skirts by the late stages, as seen in the last columns of the central and lower rows of Figure 12. Churazov et al. (2001) recognised this sensitivity, which accounts for the large skirt or secondary torus seen in their simulation, which was more prominent than any of minor rings that we obtained.

The Morganti et al. (1999) radio images of the NML show an extended region of faint emission above the bar (torus). This morphology most closely resembles that of the "young torus" with its "fuzzy hat." The height of the faint emission region is greater in comparison with the bar than we have found in our simulations (typically the simulated "fuzzy hat" reaches a maximum height that is approximately the width of the torus). The excess may 
be due to details of the initial geometry; a prolate rather than spherical initial bubble might produce a taller "hat." It may also be affected by the relative pressure between the intial bubble and the ISM. We note that the simulated radio morphologies at earlier and later stages lack extended diffuse emission above the torus. Although the comparison between the observed and simulated morphologies is not perfect, the simulation strongly suggests that the dynamical age of the NML is $t \approx 120-150 t_{0}$.

\subsubsection{A radio stalk and its motion}

The descending body of radio plasma mentioned in $\$ 4.1 .3$ at late times is a stalk on the $z$-axis. Its thickness is a few $r_{0}$, which is a fraction of the thickness of the column of slumping thermal gas carrying it. No such stalk appeared in the simulations of the M87 radio lobes by Churazov et al. (2001), which likely indicates that such fine features are sensitive to geometry and system parameters. Brüggen \& Kaiser (2001) found a radio stalk in simulations of bubbles on galaxy cluster scales, however those stalks appear when part of the bubble contracts laterally about its initial position. The stalks in our simulations involve radio plasma moving into regions previously devoid of bubble material.

In medium and late times with $\eta=10^{-2}, 10^{-3}$ (e.g. $t=600 t_{0}, 1200 t_{0}$ in Figure 12) transverse rings with diameters of $\sim 10 r_{0}$ propagate vertically up and down the stalk. It is not clear whether these features represent vertical transport of radio plasma, or a vertically propagating wave in the stalk thickness. In an off-perpendicular view the rings overlap, giving the appearance of a fat stalk with bright core and faint margins. We speculate that three-dimensional asymmetries of the rings might give qualitatively similar images at all orientations. This fat-stalk morphology has thickness comparable to the "large-scale jet" found by Morganti et al. (1999).

However, the simulated radio stalk cannot be a complete description of the "large-scale jet." The morphology of the NML alone is that of a "young torus" $\left(t \lesssim 150 t_{0}\right)$, which occurs before slumping commences in the thermal gas trunk. At this age, only uplift occurs.

The observed velocities of both the inner and outer filaments, which are respectively near the inner lobe and NML, are blue-shifted (Graham 1998; Morganti et al. 1991), indicating upflow in the regions at both ends of the "large-scale jet". We speculate that radio plasma from the inner lobe is driven by the same pressure gradient that causes the upflow, thereby achieving a similar momentum flux.

Its velocity will be higher because it is lighter with the velocity scaling approximately as the square root of the density ratio of thermal gas to radio plasma. The diameter of 
the uplifted region is determined by the extent of a low pressure region that is formed at the middle of the rising torus. Hence the diameter of the "large-scale jet" $(3 \mathrm{kpc})$ and the thermal trunk should be similar. At $t=150 t_{0}$ the width of the thermal trunk is $5.0 \mathrm{kpc}$, based upon the point where the density becomes equal to the background density. Thus the width of the trunk in the simulation and the observed width of the "large-scale jet" are similar.

Another possibility is that the jet feeding the NML is not cut off completely and that the large-scale jet represents a reduced historical level of jet activity.

\subsection{Physical implications}

\subsubsection{X-ray excess coinciding with the $N M L$}

In the "young torus" stage of our simulations, the overdense trunk of thermal gas bounded by and immediately beneath the radio plasma torus is up to a factor 2 times as dense as the undisturbed gas at equivalent locations in the potential (§2.1.1). As the $\mathrm{X}$-ray emissivity is proportional to the square of the density, the overdense trunk produces a local excess of X-ray emission.

We identify this feature with the elongated, diffuse X-ray emission coincident with the NML from $15^{\prime}$ to over $20^{\prime}$ from the nucleus, discovered by Feigelson et al. (1981).

We correct their estimate of the region's luminosity in the $0.5-4.5 \mathrm{keV}$ band, $L_{0.5-4.5 \mathrm{keV}}=$ $1 \times 10^{39} \mathrm{ergs}^{-1}$ for a distance of $3.5 \mathrm{Mpc}$ rather than the $5 \mathrm{Mpc}$ they assumed. The region has approximately the volume of a cylinder $1^{\prime}$ in diameter and $5^{\prime}$ high. Using the MAPPINGS III code $(\mathrm{v} 1.0 .0 \mathrm{~m})$ we calculated the $0.5-4.5 \mathrm{keV}$ X-ray emissivity at a temperature $0.29 \mathrm{keV}$, $2.1 \times 10^{-23}\left(n_{\mathrm{H}} / \mathrm{cm}^{-3}\right)^{2} \mathrm{ergs}^{-1} \mathrm{~cm}^{-3}$, from which we infer the number density of the thermal gas to be $n_{\mathrm{H}} \sim 1.4 \times 10^{-2} \mathrm{~cm}^{-3}$. By extrapolating the radial density profile of Forman et al. (1985), and using densities near knots of the jet derived by Schreier et al. (1981), with a correction to a temperature $k T=0.29 \mathrm{keV}$, we find that undisturbed ISM should have a density

of $\sim 1 \times 10^{-3} \mathrm{~cm}^{-3}$ at positions equivalent to the outer filaments, or $\sim 2 \times 10^{-3} \mathrm{~cm}^{-3}$ at the inner filaments. Given the above density, the cooling time of the thermal trunk is $\approx 50 \mathrm{Myr}$. Thus the gas will not cool significantly on the timescale $\approx 140 \mathrm{Myr}$ that we estimate below $(\S 4.3 .4)$.

Churazov et al. (2001) noted the connection between simulated thermal gas trunks and the elongated regions of X-ray excess trailing the radio lobes of M87. Those X-ray features appear longer in proportion to the radio lobes (lower-left panel of Figure 1 in Churazov 
et al. (2001)) than is the case for Cen A (Figure 6 of Feigelson et al. (1981)). This would be consistent with the M87 radio lobes being dynamically older than the NML of Cen A: at times of a few hundred $t_{0}$ the thermal gas trunk is proportionately longer, and the hole in the radio torus more pronounced (e.g. see our Figure 5) than at the best morphological match for Cen $\mathrm{A}\left(t \approx 150 t_{0}\right.$, Figure 4$)$; there is a lack of diffuse "fuzzy hat" radio emission above the torus. Adopting the approximate temperature of the ISM in M87 and initial bubble position of Churazov et al. (2001), the time scale of M87 is $t_{0} \approx 0.38 \mathrm{Myr}$. The preferred age of the M87 torus, $t \sim 8 \times 10^{7} \mathrm{yr}$, corresponds to $\gtrsim 210 t_{0}$, which indeed is consistent with the M87 lobe being more dynamically evolved than that of Cen A.

Our simulations yield an overdensity of $\sim 2$ times in the uplifted thermal gas trunk, relative to the undisturbed X-ray emitting atmosphere at an equivalent distance from the nucleus of the galaxy. However the ratio of the trunk to ambient densities, as calculated above from the observations of Feigelson et al. (1981); Forman et al. (1985); Schreier et al. (1981), is $\sim 7$. We note however, that in Feigelson et al. (1981) the data were not presented in such a way as to facilitate an independent estimate of the trunk density; the density of the background medium is an extrapolation. Further, high resolution Chandra observations are required for a definitive assessment. On the other hand, it is possible that bubbles that are initially larger may raise a trunk more effectively, resulting in a greater overdensity factor. We have partially confirmed in a simulation with a bubble that is larger by a factor of 1.5. This results in a maximum overdensity of 3.

\subsubsection{A nonspherical gravitational potential}

The radio structure of the NML (Figure 1) observed by Morganti et al. (1999) bends towards the north. Whereas the "large-scale jet" points approximately outwards away from the nucleus, the inferred torus is tilted by several degrees relative to the central direction and the tapering diffuse emission above it is tilted further, so that it almost points due north. We interpret this bend as evidence for a non-spherical gravitational potential. The equipotential surfaces become more prolate or oblate with distance from the nucleus, thereby providing a buoyant force that acts in different directions at different locations. This is an independent probe of the gravitational potential at large distances from the visible galaxy. 


\subsubsection{The outer filaments}

In this and the following subsections we present a model for the formation of the outer filamaents and the nearby star-formation region that utilises the velocity field in the X-ray emitting thermal gas caused by the rising torus.

The large HI cloud near the NML and outer filaments (Schiminovich et al. 1994) has a peak HI column density of $\sim 10^{21} \mathrm{~cm}^{-2}$ and typical width $\sim 3 \mathrm{kpc}$, implying an average number density $n_{\mathrm{H}} \sim 0.1 \mathrm{~cm}^{-3}$. Local regions may have densities greater or lesser than the average. The HI cloud has a blueshift of $\sim 200 \mathrm{~km} \mathrm{~s}^{-1}$ with respect to the systemic velocity of the galaxy Schiminovich et al. (1994).

In projection, the eastern edge of the cloud is adjacent to the "large-scale jet" (Morganti et al. 1999), the region of young stars and the outer filaments (Graham 1998; Mould et al. 2000). Thus, the large cloud appears to have entered the region affected by the overdense region affected by the rising torus of the NML. Gas on the disturbed edge of the cloud is shock-compressed to form stars (\$4.3.4) and the flow also ablates gas from the edge. These initially cold and dense clouds are accelerated and shredded by the Kelvin-Helmholtz and Rayleigh-Taylor instabilities in a cascading process that results in a stream of small cloudlets that are eventually accelerated to an appreciable fraction of the free-stream velocity.

The ablated clouds initially move at the orbital velocity of the large HI cloud, (i.e. are blusehifted with a velocity $\sim 200 \mathrm{~km} \mathrm{~s}^{-1}$ ) approximately perpendicular to the X-ray gas upflow. The ram-pressure acceleration of clouds by the upflow increases the velocity dispersion. This is consistent with the velocity dispersion of order the atmospheric sound speed (Graham 1998). For a spherical cloud with a density of $\rho_{c}$ and a radius of $R_{c}$ driven by a medium with a density of $\rho_{a}$ moving at a velocity $V_{a}$, the drag force is $F_{D}=C_{D} \pi R_{c}^{2} \rho_{a} V^{2}=$ $(4 \pi / 3) R_{c}^{3} \rho_{c}\left(d v_{c} / d t\right)$ where $C_{D} \sim 1$ is a drag coefficient. Thus the acceleration timescale, $t_{\mathrm{acc}} \approx V\left(d v_{c} / d t\right)^{-1}$ is approximately

$$
t_{\mathrm{acc}} \approx 5.4 \times 10^{4} \mathrm{yr} \frac{\rho_{\mathrm{c}}}{\rho_{\mathrm{a}} C_{D}}\left(\frac{R_{c}}{20 \mathrm{pc}}\right)\left(\frac{V_{a}}{270 \mathrm{~km} \mathrm{~s}^{-1}}\right)^{-1} .
$$

The streaming X-ray emitting gas has a density $n_{a} \sim 10^{-2} \mathrm{~cm}^{-3}$ (Feigelson et al. 1981) (see §4.3.1). In observations of the filaments, Morganti et al. (1991) measured the [SII] doublet to be in the low-density limit, implying $n_{c}<10^{2} \mathrm{~cm}^{-3}$, which is not diagnostic. The density of the large HI cloud, as the source of the small clouds, is likely to be give a more realistic value of the cloud density, $n_{c} \sim 0.1 \mathrm{~cm}^{-3}$.

The total pressure (static plus ram) of the stream drives a shock into each cloud. (The HI cloud may be underpressured with respect to the stream, so that we incorporate the effect 
of the static pressure.) Let $c_{I}$ be the isothermal sound speed of the stream and $v_{\text {rel }}$ be the relative velocity between the moving cloud and the stream. Then, the velocity of the cloud shock is:

$$
\begin{aligned}
v_{\mathrm{sh}} & \approx \sqrt{\frac{2}{\gamma+1} \frac{P_{\mathrm{a}}+\rho_{\mathrm{a}} v_{\mathrm{rel}}^{2}}{\rho_{\mathrm{c}}}}=\sqrt{\frac{2}{\gamma+1} \frac{\rho_{\mathrm{a}}}{\rho_{\mathrm{c}}}\left(c_{I}^{2}+v_{\mathrm{rel}}^{2}\right)} \\
& =110\left(\frac{n_{\mathrm{a}}}{0.01 \mathrm{~cm}^{-3}}\right)^{1 / 2}\left(\frac{n_{\mathrm{c}}}{0.1 \mathrm{~cm}^{-3}}\right)^{-1 / 2} \frac{\sqrt{c_{I}^{2}+v_{\mathrm{rel}}^{2}}}{400 \mathrm{~km} \mathrm{~s}^{-1}} \mathrm{~km} \mathrm{~s}^{-1}
\end{aligned}
$$

If the cloud has no component of velocity in the direction of the stream then $v_{\text {rel }} \approx\left(270^{2}+\right.$ $\left.200^{2}\right)^{1 / 2} \approx 340 \mathrm{~km} \mathrm{~s}^{-1}$; for an ambient temperature $k T \approx 0.29 \mathrm{keV}, c_{I} \approx 210 \mathrm{~km} \mathrm{~s}^{-1}$ and $\left(c_{I}^{2}+v_{\text {rel }}^{2}\right)^{1 / 2} \approx 400 \mathrm{~km} \mathrm{~s}^{-1}$. The shock velocity decreases for larger cloud densities and increases if there is a cloud velocity component towards the nucleus.

The timescale for shredding of a cloud is usually shorter than the acceleration timescale. Let the diameter of a cloud in the flow be $D_{\mathrm{c}}$ and the velocity of the cloud shock be $v_{\mathrm{sh}}$, then the shredding timescale of a shocked cloud in the upflow is $t_{\text {shred }} \sim D_{\mathrm{c}} / v_{\mathrm{sh}} \approx 2 \times$ $10^{5}\left(D_{\mathrm{c}} / 20 \mathrm{pc}\right)\left(v_{\mathrm{sh}} / 100 \mathrm{~km} \mathrm{~s}^{-1}\right)^{-1} \mathrm{yr}$ (Klein et al. 1994). As a result of the sheredding, the acceleration time of the clouds becomes progressively shorter. If the dense cloudlets have a fractal structure and a distribution of sizes then the clouds will cover a range of velocities and degrees of shredding. The outer filaments do indeed show such a range of morphologies - from large arcs to elongated smears distributed in the direction away from the galaxy nucleus (Morganti et al. 1991; Graham 1998). We attrribute the former to clouds in the initial stages of acceleration; in this case the shapes of the emission line arcs trace the outline of the cloud. We attribute the smeared features to the shredded remnants of previously accelerated clouds. At the available resolution in the images of Morganti et al. (1991); Graham (1998), the smallest visible knots are $\sim 1-2^{\prime \prime}$ wide, corresponding to a radial scale $R_{c} \sim 10-20$ pc.

We now consider some of the features in the outer filament region that enable us to estimate the density and cloud shock velocities. The largest distinct structures amongst the outer filaments are arcs with $\sim 70$ pc radii of curvature (Morganti et al. 1991; Graham 1998). By integrating the [OIII] surface brightness contours in Morganti et al. (1991) the luminosity of the prominent arc at the southern end of the outer filaments is found to be $L_{[\mathrm{OIII}]} \approx(1.3-2.6) \times 10^{36} \mathrm{ergs}^{-1}$. The filaments' $[\mathrm{OIII}] / \mathrm{H} \beta$ ratios $\sim 3-5$ (Graham 1998) imply shock velocities $\sim 80 \lesssim v_{\text {shock }} \lesssim 120 \mathrm{~km} \mathrm{~s}^{-1}$. Assuming spherical cap-like geometry for this arc then the shock surface area of this arc $\approx 2.5 \times 10^{41} \mathrm{~cm}^{2}$. We used MAPPINGS III to calculate [OIII] emissivities for shock velocities ranging from $80-120 \mathrm{~km} \mathrm{~s}^{-1}$, to infer a number density $n_{\mathrm{H}} \approx 0.3-0.9 \mathrm{~cm}^{-3}$ in the cloud. This number density would be less if the shock were corrugated. Densities at the lower end of this range are consistent with 
those required for shock velocities greater than $80 \mathrm{~km} \mathrm{~s}^{-1}$, this being the threshold value for [OIII]emission.

On the other hand, as we have indicated in (4.3.1), the present simulations may not have been successful in reproducing the observed over-density of uplifted thermal gas. We have indicated that this may be related to the intial size of the NML precursor. On the basis of the current simulations, therfore, we cannot exclude the possibility that the ram pressure of the "large-scale jet" may play a role in exciting the outer filaments. Future X-ray observations are crucial to better constraining the density of hot gas in the vicinity of the NML.

With densities $n_{c} \sim 0.1 \mathrm{~cm}^{-3}$ and $n_{a} \sim 1.4 \times 10^{-2} \mathrm{~cm}^{-3}$, the acceleration timescales of clouds with radius $70 \mathrm{pc}$ are less than $2 \mathrm{Myr}$. The timescale for disruption of a $70 \mathrm{pc}$-radius cloud is $\sim 1.5 \mathrm{Myr}$. The similarity of these timescales is consistent with a distribution of cloud morphologies from large, nearly round blobs to small, smeared filamentary streaks (see images in Morganti et al. 1991; Graham 1998).

Thus the spatial and velocity distribution of the outer filaments appears consistent with the time and length scales of cloudlets in the HI region being swept into the upflow of X-ray emitting gas beneath the NML torus. The pressure of the stream on the faces of the largest blobs may account for their excitation.

The inner filaments may be undergoing the same processes as the outer filaments. It is noteworthy that the velocity spread within each component of the inner filaments is transonic in the X-ray emitting gas, $\lesssim 270 \mathrm{~km} \mathrm{~s}^{-1}$. However there must be additional influences because the spread of velocities in the overall inner filament region is approximately $360 \mathrm{~km} \mathrm{~s}^{-1}$ (Morganti et al. 1991), which exceeds the sound speed of a $k T=0.29 \mathrm{keV}$ gas. The detailed hydrodynamics of this region may be affected by the "large-scale jet" and the inner lobe, and this is beyond the scope of the present paper.

\subsubsection{Star formation, merger history, \&5 the AGN source}

With a length scale $r_{0} \approx 0.2 \mathrm{kpc}$ (in accordance with the height of the inferred torus in the NML, see Figures 8, 10), and with an ISM gas temperature of $k T=0.29 \mathrm{keV}$, the morphologically inferred bubble age of $t$ approx $150 t_{0}$, corresponds to $1.4 \times 10^{2} \mathrm{Myr}$, using the value of $t_{0} \equiv r_{0} / v_{0}$ given by equation (3-11). At a time, $t=150 t_{0}$, the velocity of rise of both the $\eta=10^{-2}$ and $10^{-3}$ bubbles is $0.27 v_{0} \approx 60 \mathrm{~km} \mathrm{~s}^{-1}$ (see figure 8). The time of transit past the $\sim 1 \mathrm{kpc}$ region occupied by the Graham (1998) field of the outer filaments is therefore $\approx 16 \mathrm{Myr}$, exactly the age of the young stars estimated by Mould et al. (2000). This strongly reinforces a dynamical connection between the NML and the formation of the 
stars.

In our model, star formation is induced by shocks resulting from the impact of the X-ray gas on the HI cloud adjacent to the NML (e.g. Schiminovich et al. 1994). The growth time and length scales of a maximally unstable post-shock region are estimated in equations (9) - (11) in Bicknell et al. (2000) on the basis of the theory of Elmegreen \& Elmegreen (1978). For an external gas density, $n_{\mathrm{H} \text {,ext }} \sim 10^{-2} \mathrm{~cm}^{-3}$, and a cloud density, $n_{\mathrm{H}} \mathrm{cm}^{-3}$ the shock velocity in the cloud (4-2) is $v_{\text {shock }} \approx 35 n_{\mathrm{H}}^{-1 / 2} \mathrm{~km} \mathrm{~s}^{-1}$, The time scale for maximal growth is $t_{\mathrm{mgr}} \sim 6 n_{\mathrm{H}}^{-1 / 2} \mathrm{Myr}$. After a time of $t_{6} \mathrm{Myr}$, the maximally growing wavelength, $\lambda_{\mathrm{mgr}} \sim 4 n_{\mathrm{H}}^{1 / 2} t_{6} \mathrm{pc}$ is well within the scale of the star-forming region. Thus, consistent with the observations of Mould et al. (2000), significant star formation can occur within a few Myr, especially if $n_{\mathrm{H}}>0.1$. This suggests that the emission line region is adjacent to a star-forming region with somewhat greater density.

The estimate of the age of the NML $\sim 1.4 \times 10^{2} \mathrm{Myr}$ is significant. We suggest that this corresponds to the disruption of radio activity within Cen $\mathrm{A}$ as a result of the merger that created the present dust lane. The detachment of the NML precursor from the presently growing inner lobe implies that jet activity was absent or greatly reduced during an interval $\sim 10^{2}$ Myr. The duration of this interruption is on the order of a dynamical time-scale of gas within galaxy. Moreover this time-scale is typical of the proposed duration of the merger of a spiral galaxy into NGC 5128, and the subsequent evolution of the warped dust-lane disk. Tubbs (1980) performed test-particle kinematic studies with an initially flat disk warping due to differential precession in a prolate galaxian potential. The presently observed disk warp was matched at a simulated age of $2.3 \times 10^{2} \mathrm{Myr}$ (after correcting the galaxy's distance to $3.5 \mathrm{Mpc}$ ). However in further geometric models of initially flat disks, comprised of mutuallygravitating rings, in an oblate potential, Sparke (1996) determined an age $\sim 1$ Gyr. Quillen et al. (1993) considered the infall and tidal stripping of a spiral galaxy, starting at a time when the spiral galaxy was $15^{\prime}$ from the nucleus of NGC 5128. They found that a non-flat disk could be produced directly due to the varying angular momentum of the tidally stripped material, and that the present disk morphology can occur as early as $1.6 \times 10^{2} \mathrm{Myr}$ after the start of the merger. This latter scenario is most consistent with our age for the NML.

We speculate that the jet was disrupted by the merger event and that a large flow of gas to the nucleus on the merger timescale and either interrupted the accretion flow into the nucleus or obstructed the jets out of the nucleus. The time elapsed between the detachment of the NML precursor and the formation of the new inner lobe supports the model of Quillen et al. (1993). 


\section{DISCUSSION AND CONCLUSIONS}

Following the simulations of buoyant radio-emitting bubbles by Churazov et al. (2001) and Brüggen \& Kaiser (2001), we have investigated the relevance of such a model to the Northern Middle Lobe of Centaurus A, examining the hypothesis that it originated as a radio lobe created in an earlier episode of jet activity. In so doing we have extended these papers in the following ways:

- We have used an ISM model appropriate for Cen A and examined the importance of the $\beta$ parameter on the dynamics of the bubble.

- We have used an initial geometry for the bubble that is typical of the inner radio lobe of Cen A, as a precursor to the present middle lobe, albeit a factor of two larger in size and distance from the nucleus. (Churazov et al. (2001) used a bubble over twice as large in comparison to its displacement from the nucleus, whereas Brüggen \& Kaiser (2001) studied a bubbles starting within a core radius away from the origin of the potential).

- We have examined in detail the effect of the density contrast between the radio bubble and the ambient medium, for values of the density contrast, $\eta$, as low as $10^{-3}$. We found that some aspects of the bubble evolution are sensitive to the difference between $\eta=10^{-2}$ and $\eta=10^{-3}$. For example, lateral spreading of the torus (Figure 9) and the time at which the maximum convection velocity occurs (Figure 7 ).

In the light of these simulations, we have proposed a model in which the precursor to the northern middle lobe of Centaurus A grew in an episode of jet activity which ceased some time ago. At the time when the jet feeding the lobe was interrupted, the lobe was approximately twice as large and twice as distant from the nucleus as the present day inner lobe. Since then it has been rising buoyantly away from the nucleus. (with a density contrast

$\eta<10^{-2}$ relative to its surroundings). This model explains the gross features of the NML, namely its bright base and the fainter radio structure above it. A more detailed comparison with model and observation would require fine-tuning of the initial parameters. For example, one could investigate an elliptical configuration for the initial structure of the NML or the effects of an elliptical potential. Nevertheless, comparison of the gross features of the radio morphology with the simulations is sufficient for us to date the NML at $\sim 1.4 \times 10^{2} \mathrm{Myr}$. We have suggested that the time of interruption of jet activity corresponds to the time at which the often-discussed merger took place in Centaurus A and we regard the Quillen et al. (1993) estimate of $1.6 \times 10^{2} \mathrm{Myr}$ for the time it has taken the NGC 5128 disk to settle into its present configuration as compelling confirmation of this idea. This is significant evidence 
that mergers can disrupt existing AGN activity, possibly by their effect on the mass transfer to the nucleus. If a kpc-scale accretion flow is disrupted in this way, then it takes some time (a few orbital timescales) for the gas that has been captured by the galaxy to settle again into a steady accretion flow. Alternatively, if the jet is smothered by clouds of material accreted from the consumed disk galaxy then a similar timescale may elapse before the matter settles away from the jet axis. We suggest that this has occurred in Centaurus A, leading to the renewed onset of radio activity represented by the inner lobe and jet.

This conclusion may be related to other observations of young radio galaxies and may well provide a fresh way of looking at the relationship between mergers and radio activity. For example Perlman et al. (2001) have found evidence for old ( $\left.\sim 10^{8} \mathrm{yr}\right)$ merging activity in three young Compact Symmetric Object (CSO) radio galaxies and have suggested that the mergers may be related to a delayed onset of radio activity. The parallels with Centaurus A are obvious.

The bubble model explains the gross features in radio and X-rays on the scale of the middle and inner radio lobes. The rising bubble is surrounded by a transonic circulation in the denser, ambient, X-ray emitting medium. The vortex ring pattern is substantially larger than the extent of radio-emitting plasma and follows the bubble as it rises. Wisps of radio-emitting plasma are drawn off the bubble by the circulating gas and extend high above the main part of the bubble at the present stage. A trunk of overdense X-ray emitting gas is drawn up from below the bubble, deforming it into a torus. The overdense trunk is identified with the diffuse X-ray excess coinciding with the NML discovered by Feigelson et al. (1981). Although the ensuing phase of renewed jet activity is not addressed by our simulations we have suggested that the pressure gradient in the upflow region beneath the NML drew light radio plasma from the top of the newly forming inner lobe. This is our suggestion for the "large-scale jet" crossing the gap between the inner and middle radio lobes. Nevertheless, this suggestion remains to be confirmed by future simulations involving two lobes.

In our model, the evolution of the buoyant radio-plasma bubble on kpc scales also accounts for features observed on scales of tens of parsecs or less. About $\sim 16 \mathrm{Myr}$ ago the convection flow caused by the bubble interacted with a massive HI cloud, resulting in both shock-induced star formation and ablation of gas from the periphery of the cloud. Thus we have proposed that the outer filaments of emission line gas are clouds of a few pc to $\sim 150 \mathrm{pc}$ thickness swept into the transonic flow and shock-excited by the dynamic pressure of the stream. The density and shock velocity estimates are reasonably consistent here with the proviso that the estimated density be used. In this context we have noted the importance of new X-ray observations of the NML of Centaurus A and the need for further simulations with different geometry. 
The velocity dispersion in the ablated clouds results from their different sizes and different times of ablation. The inner filaments may also be affected by the thermal upflow. However, their large velocity spread $\sim 360 \mathrm{~km} \mathrm{~s}^{-1}$ is not reproduced by our simulations

motivating further work involving the effect of the initial geometry on the structure of the flow.

\section{ACKNOWLEDGEMENTS}

We thank R. Morganti for the radio image of the northern middle lobe of Centaurus A. This work was supported by an Australian Research Council Large Grant, A69905341

\section{REFERENCES}

Bicknell, G. V., Sutherland, R. S., van Breugel, W. J. M., Dopita, M. A., Dey, A., \& Miley, G. K. 2000, ApJ, 540, 678

Bicknell, G. V. 1995, ApJS, 101, 29

Blanco, V. M., Graham, J. A., Lasker, B. M., \& Osmer, P. S. 1975, ApJ, 198, L63

Bland, J., Taylor, K., \& Atherton, P. D. 1987, MNRAS, 228, 595

Brüggen, M. \& Kaiser, C. R. 2001, MNRAS, 325, 676

Churazov, E., Brüggen, M., Kaiser, C. R., Böhringer, H., \& Forman, W. 2001, ApJ, 554, 261

Colella, P. \& Woodward, P. R. 1984, J. Comput. Phys., 54, 174

Cooper, B. F. C., Price, R. M., \& Cole, D. 1965, Aust. J. Phys., 18, 589

Elmegreen, B. G. \& Elmegreen, D. M. 1978, ApJ, 220, 1051

Feigelson, E. D., Schreier, E. J., Delvaille, J. P., \& Giacconi, R. 1981, ApJ, 251, 31

Forman, W., Jones, C., \& Tucker, W. 1985, ApJ, 293, 102

Graham, J. A. \& Price, R. M. 1981, ApJ, 247, 813

Graham, J. A. 1998, ApJ, 502, 245 
Hui, X., Ford, H. C., Freeman, K. C., \& Dopita, M. A. 1995, ApJ, 449, 592

Jones, D. L., et al. 1996, ApJ, 466, L63

Killeen, N. E. B. \& Bicknell, G. V. 1988, ApJ, 325, 165

Klein, R. I., McKee, C. F., \& Colella, P. 1994, ApJ, 420, 213

Lauer, T. R., et al. 1995, AJ, 110, 2622

Malin, D. F., Quinn, P. J., \& Graham, J. A. 1983, ApJ, 272, L5

Marconi, A., Schreier, E. J., Koekemoer, A., Capetti, A., Axon, D., Macchetto, D., \& Caon, N. 2000, ApJ, 528, 276

Mathieu, A., Dejonghe, H., \& Hui, X. 1996, A\&A, 309, 30

Morganti, R., Killenn, N. E. B., Ekers, R. D., \& Oosterloo, T. A. 1999, MNRAS, 307, 750

Morganti, R., Robinson, A., Fosbury, R. A. E., di Serego Alighieri, S., Tadhunter, C., \& Malin, D. 1991, MNRAS, 249, 91

Mould, J. R., et al. 2000, ApJ, 536, 266

Nicholson, R. A., Bland-Hawthorn, J., \& Taylor, K. 1992, ApJ, 387, 503

Nulsen, P. E. J. \& Böhringer, H. 1995, MNRAS, 274, 1093

Owen, F. N., Eilek, J. A., \& Kassim, N. E. 2000, ApJ, 543, 611

Perlman, E. S., Stocke, J. T., Conway, J., \& Reynolds, C. 2001, astro-ph/, 0104439, 000

Quillen, A. C., Zeeuw, de P. T., Phinney, E. S., \& Phillips, T. G. 1992, ApJ, 391, 121

Quillen, A. C., Graham, J. R., \& Frogel, J. A. 1993, ApJ, 412, 550

Richter, O. G., Sackett, P. D., \& Sparke, L. S. 1994, AJ, 107, 99

Schiminovich, D., van Gorkom, J. H., van der Hulst, J. M., \& Kasow, S. 1994, ApJ, 423, L101

Schreier, E. J., Burns, J. O., \& Feigelson, E. D. 1981, ApJ, 251, 523-529

Soria, R., et al. 1996, ApJ, 465, 79

Sparke, L. S. 1996, ApJ, 473, 810 
Sutherland, R. S., Bicknell, G. V., \& Dopita, M. A. 1993, ApJ, 414, 510

Sutherland, R. S., Bisset, D. K., \& Bicknell, G. V. 2001, ApJ, submitted,

Tingay, S. J., Jauncey, D. L., \& Reynolds, J. E. 1998, AJ, 115, 960

Tubbs, A. D. 1980, ApJ, 241, 969

Turner, T. J., George, I. M., Mushotzky, R. F., \& Nandra, K. 1997, ApJ, 475, 118

Wagner, S. J., Döbereiner, S., \& Junkes, N. 1996, MPE Report, 263, 521 


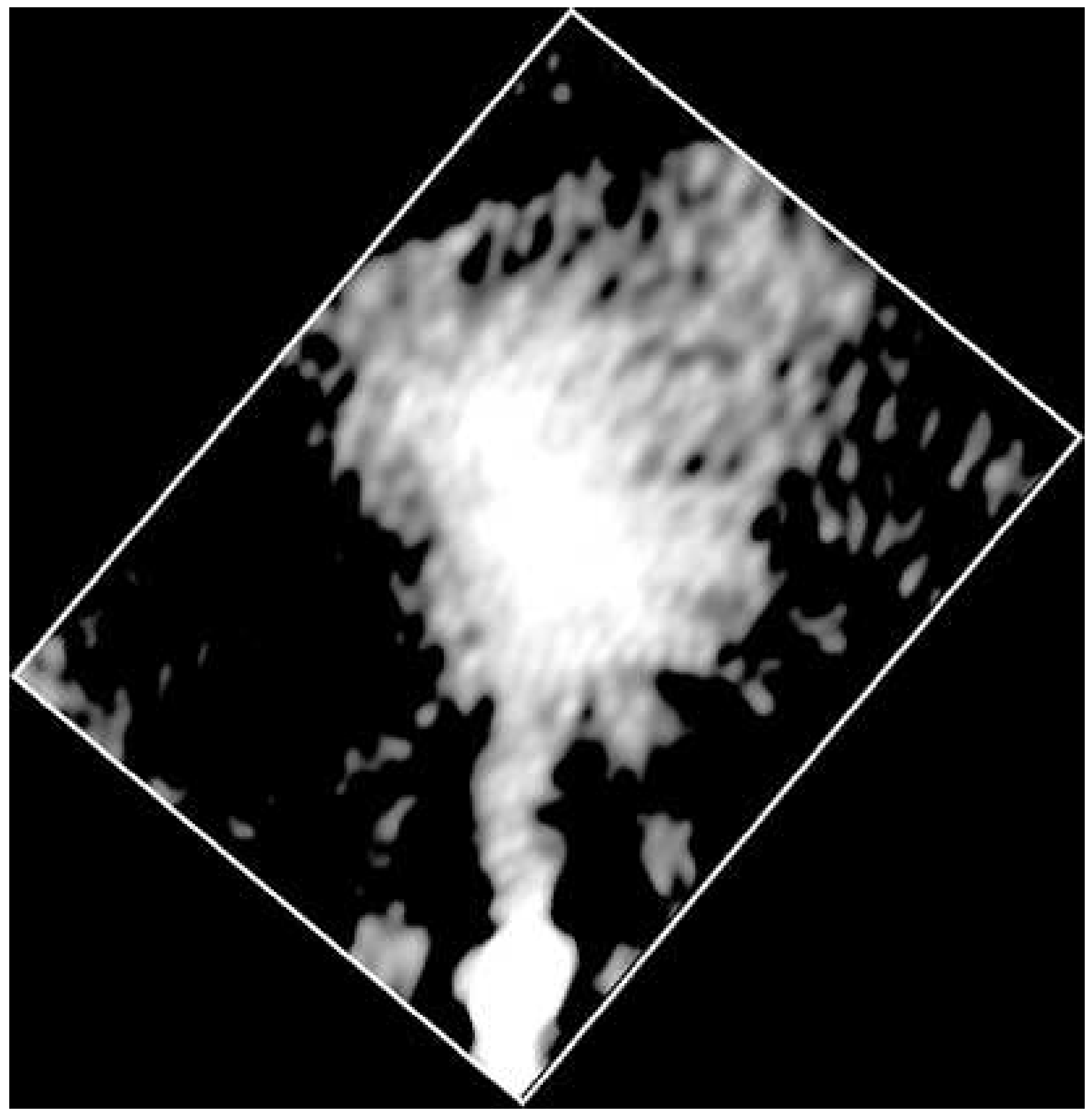

Fig. 1. - Logarithmic radio image of the Cen A corresponding to Figure 2 of Morganti et al. (1999). The inner lobe (bottom) is connected to the NML through a "large-scale jet." Sides of the white box are aligned with the RA and DEC axes; the image is rotated so that the axis of the "large-scale jet" is upwards. By permission of Dr R. Morganti. 


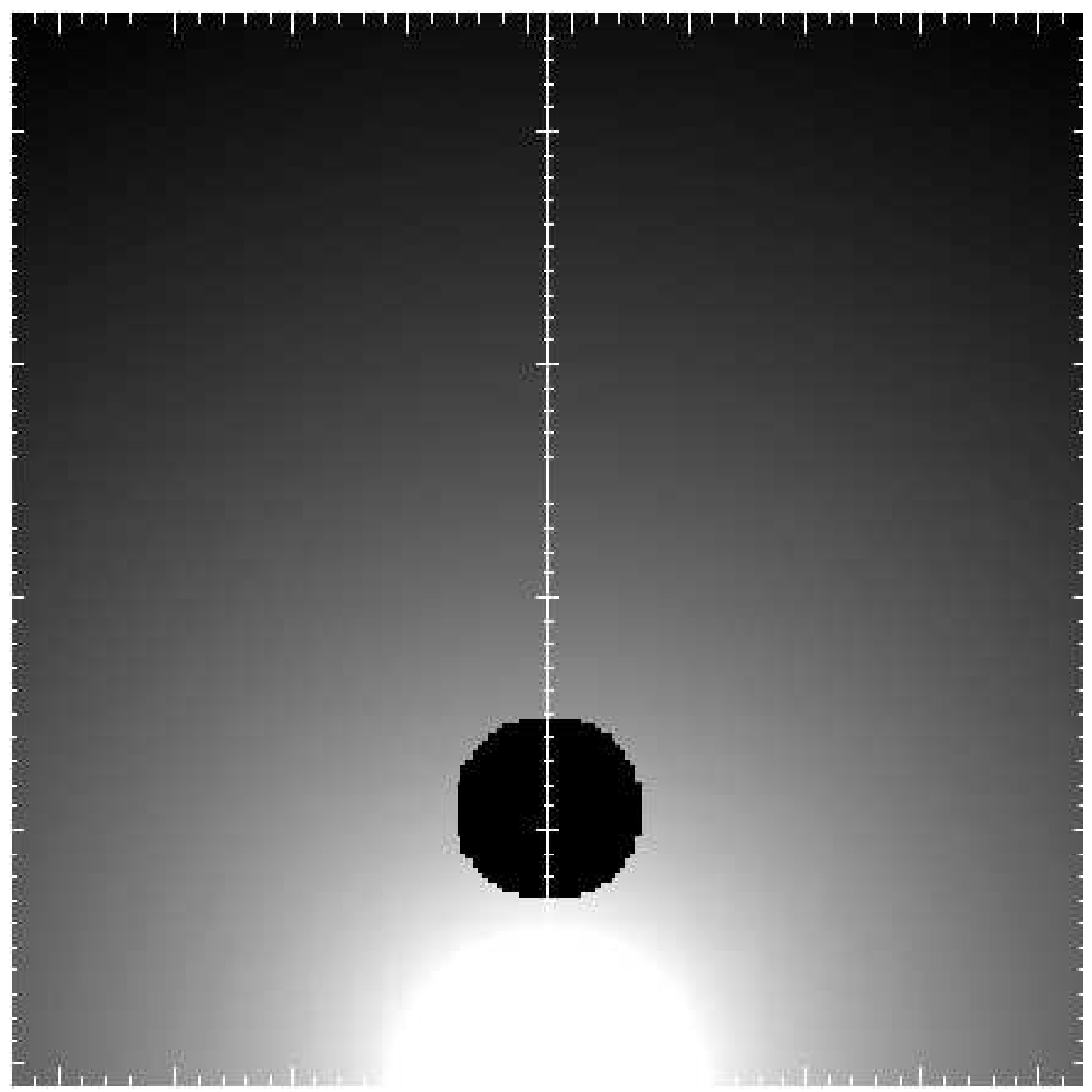

Fig. 2. - Initial density field of the $\eta=10^{-3}$ simulation, with triple the standard spatial resolution. Greyscale brightness is proportional to $\log _{10} \rho$, with saturation at a fixed level near the nucleus. The subregion shown here has dimensions $140 \times 70$ pixels, $\left(\right.$ or $\approx 233 r_{0} \times$ $\left.117 r_{0}\right)$ in the cylindrical $z$ and $r$ directions. The $z$ axis is directed upwards; the galaxy nucleus is at the origin, at the bottom edge of the frame. 


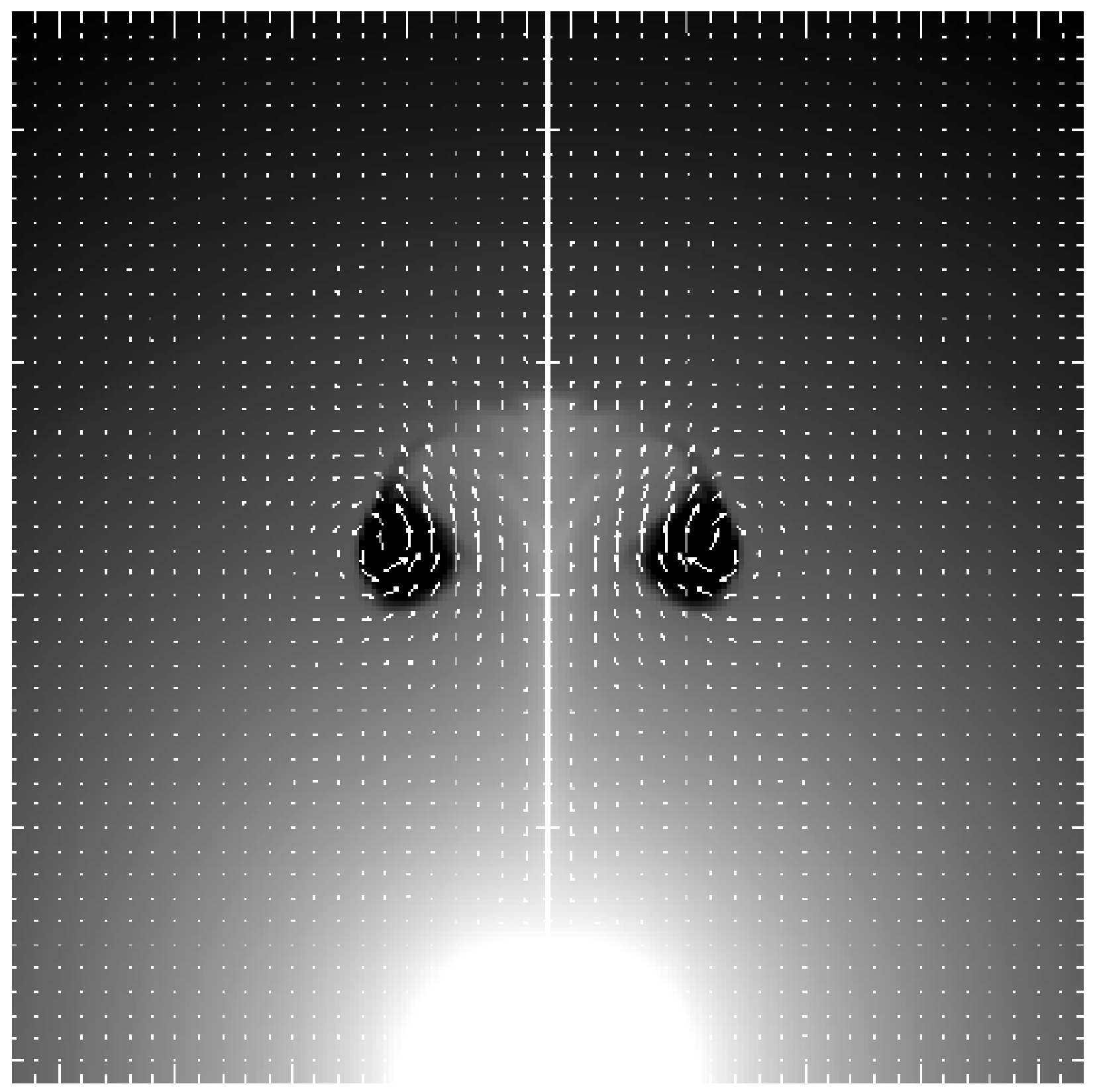

Fig. 3. - Flow velocity vectors at time $t=60 t_{0}$ in the $\eta=10^{-3}$ simulation with triple the standard spatial resolution. The maximum velocity is $\approx 1.2 v_{0}$. The region and density scale are the same as in Figure 2. The thermal gas trunk has almost broken through the initial bubble; a shock has propagated outwards from the initial shock position and appears as a circular density discontinuity. 


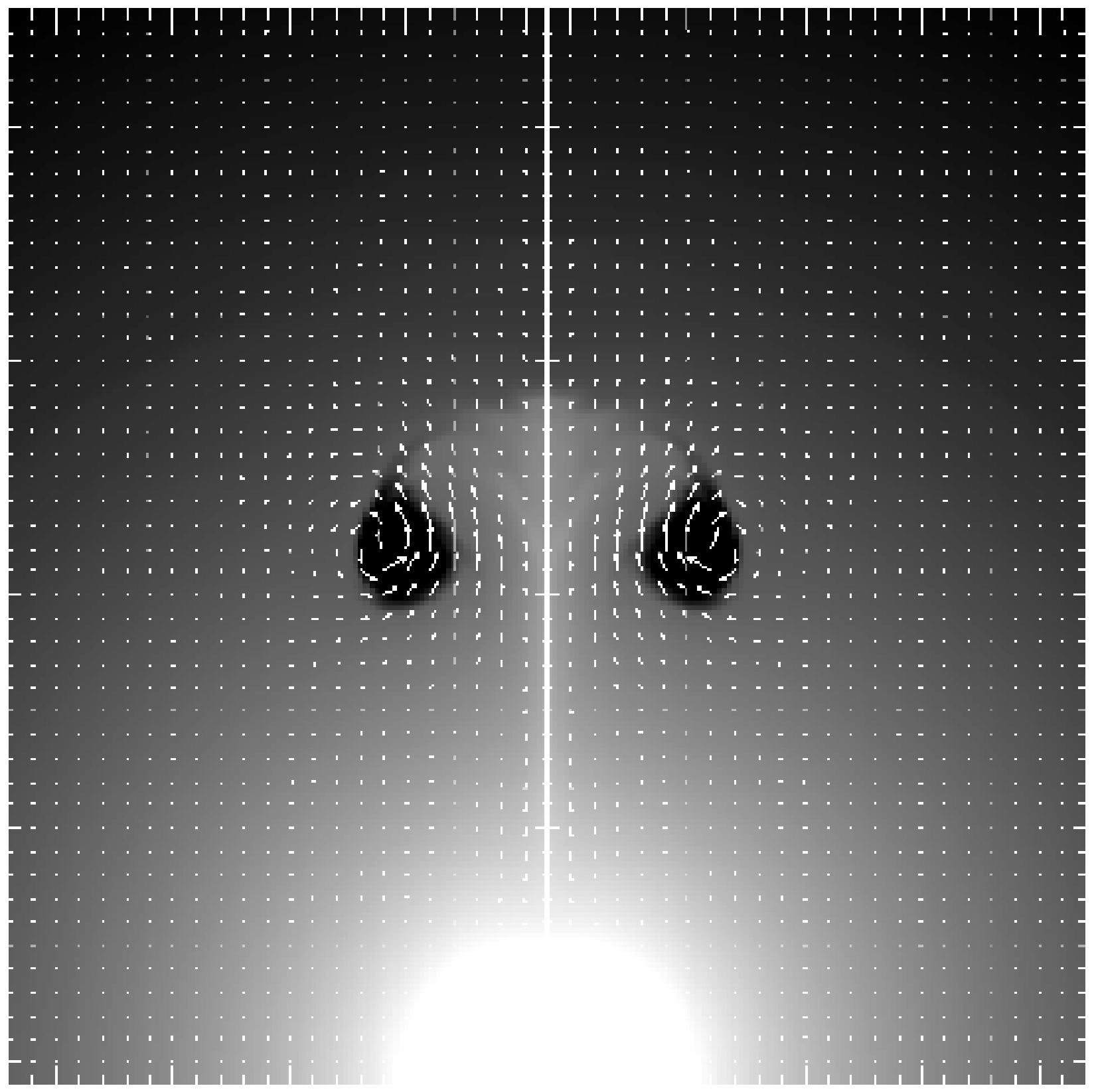

Fig. 4. - Flow velocity vectors at time $t=150 t_{0}$ in the $\eta=10^{-3}$ simulation with triple the standard spatial resolution. This is the approximate time when the simulated radio morphology matches observations. The maximum velocity is $\approx 1.2 v_{0}$. The region and density scale are the same as in Figure 2. 


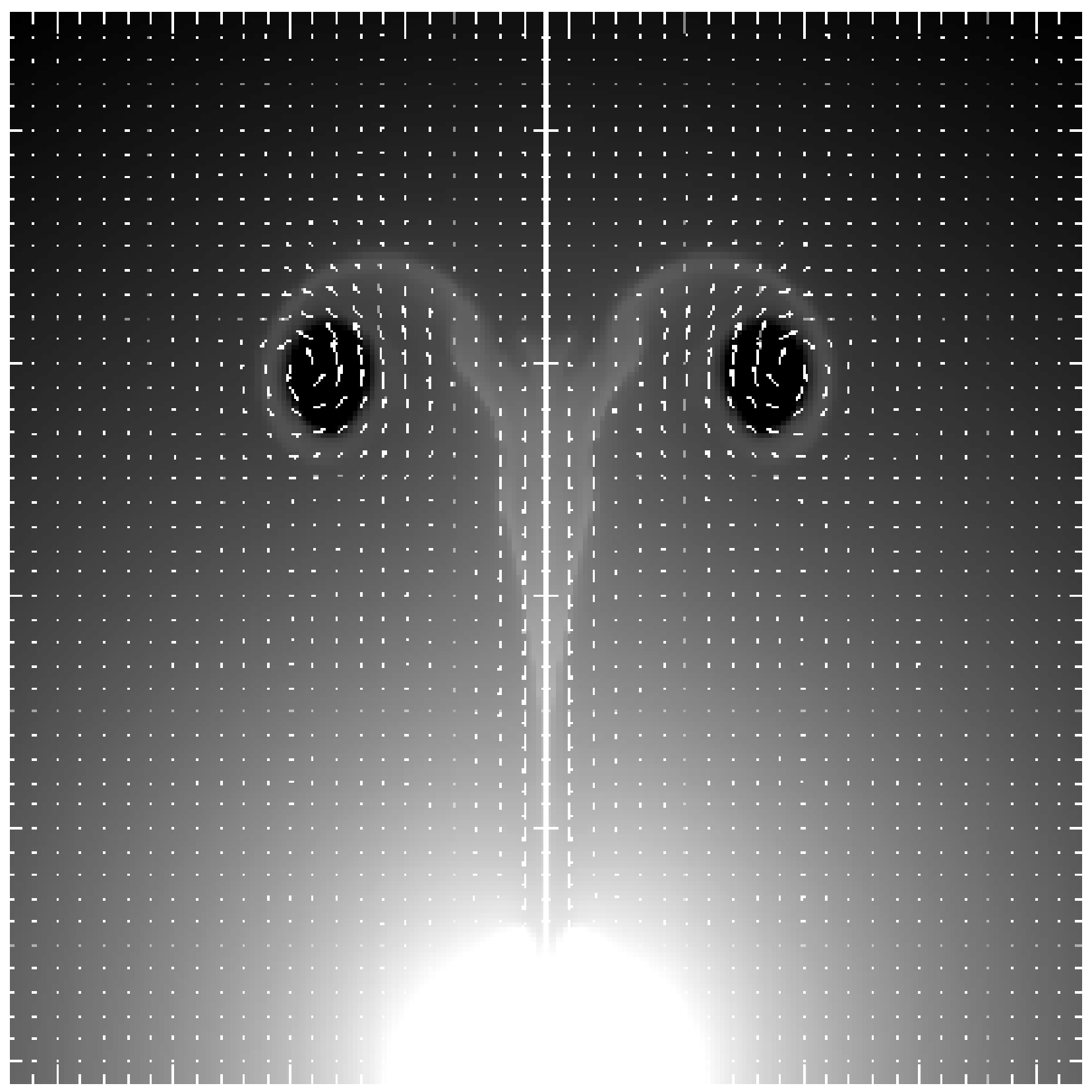

Fig. 5. - Flow velocity vectors at time $t=300 t_{0}$ in the $\eta=10^{-3}$ simulation with triple the standard spatial resolution. The maximum velocity is $\approx 1.0 v_{0}$. The region and density scale are the same as in Figure 2. 


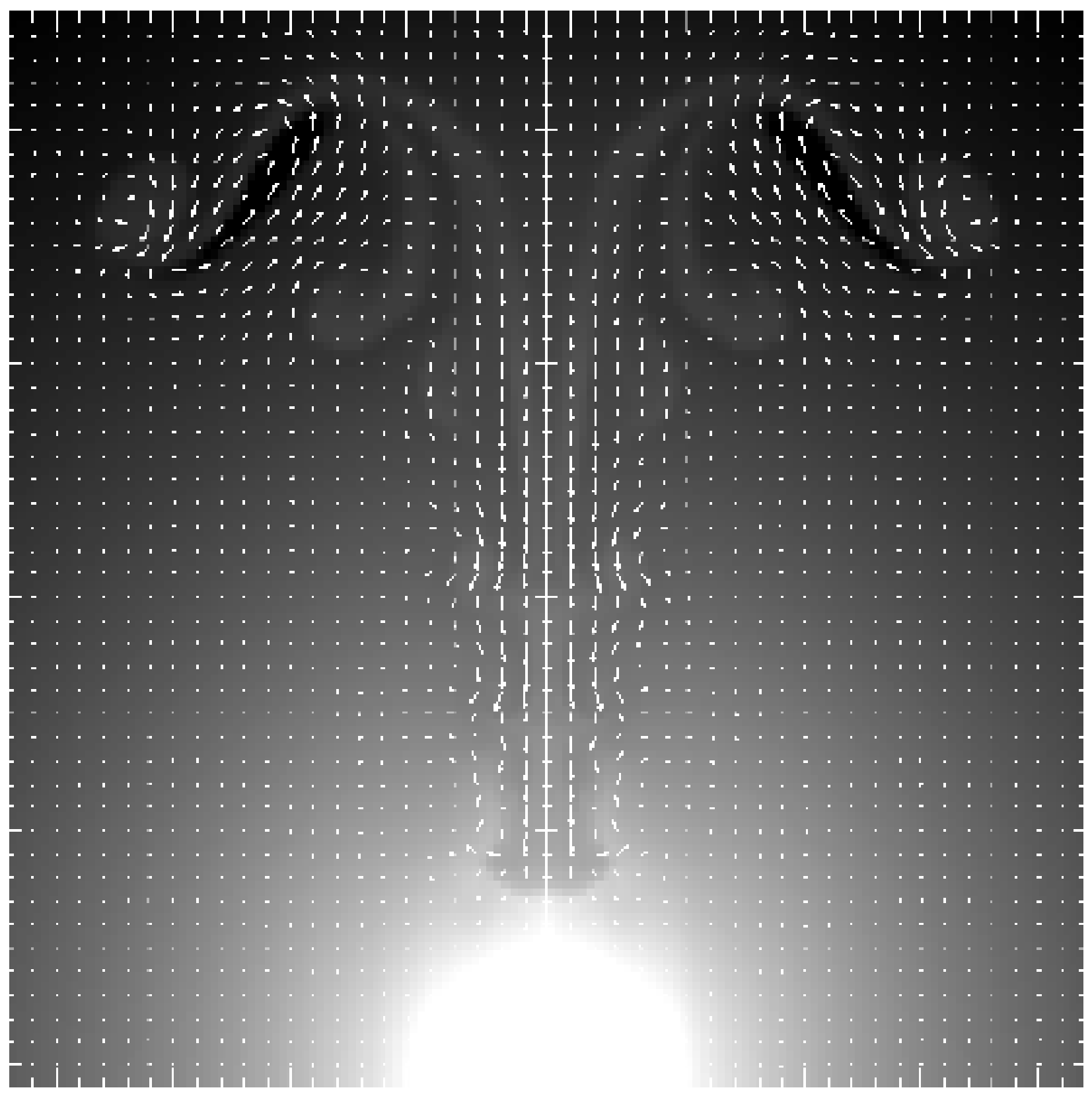

Fig. 6. - Flow velocity vectors at time $t=600 t_{0}$ in the $\eta=10^{-3}$ simulation with standard spatial resolution. The maximum velocity is $\approx 0.7 v_{0}$. The region and density scale are the same as in Figure 2. 


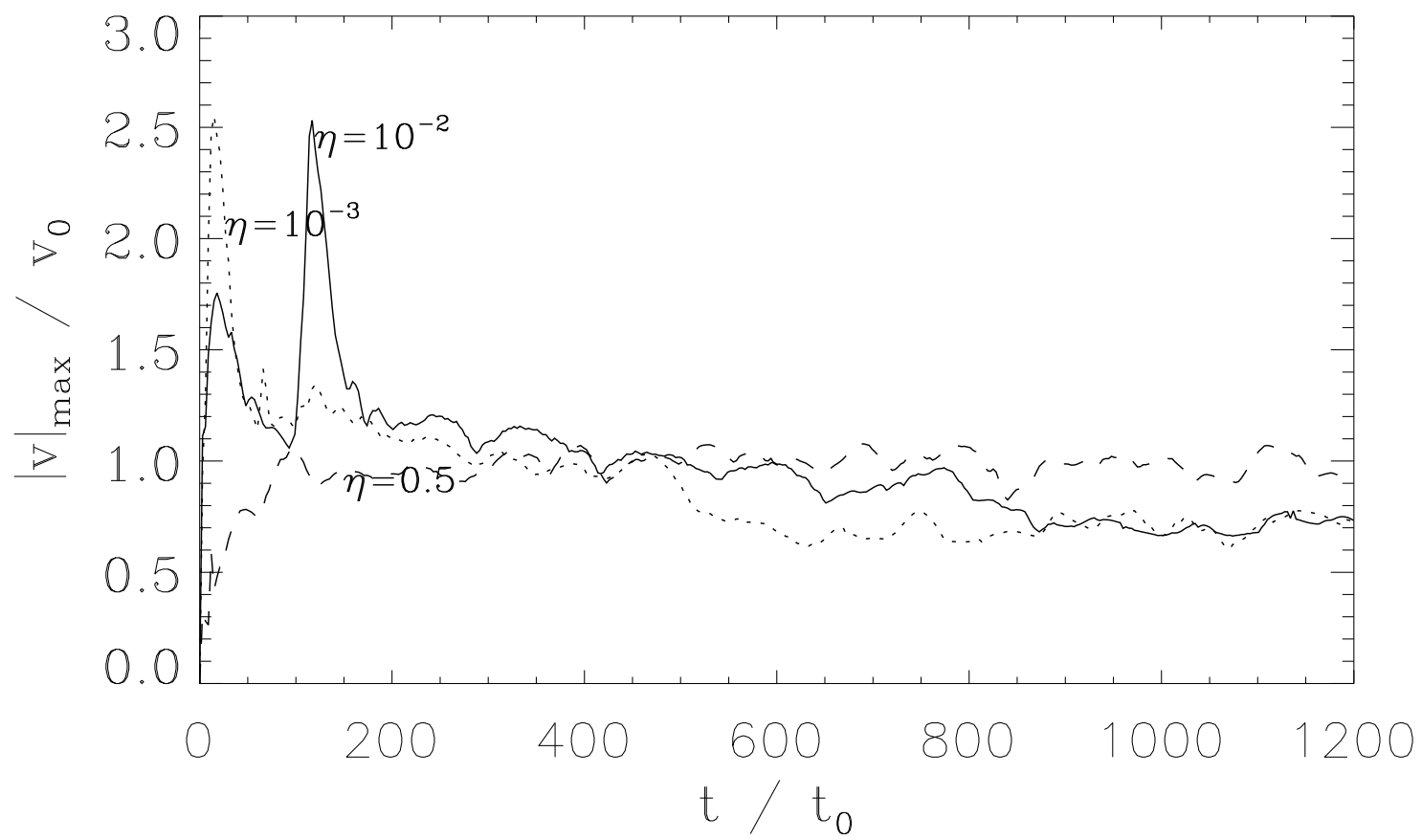

Fig. 7.- Maximum values of the velocity as a function of time, for cases $\eta=0.5,10^{-2}, 10^{-3}$ (dashed, solid and dotted lines respectively). The adiabatic sound speed in the undisturbed gas is $\approx 1.29$. 


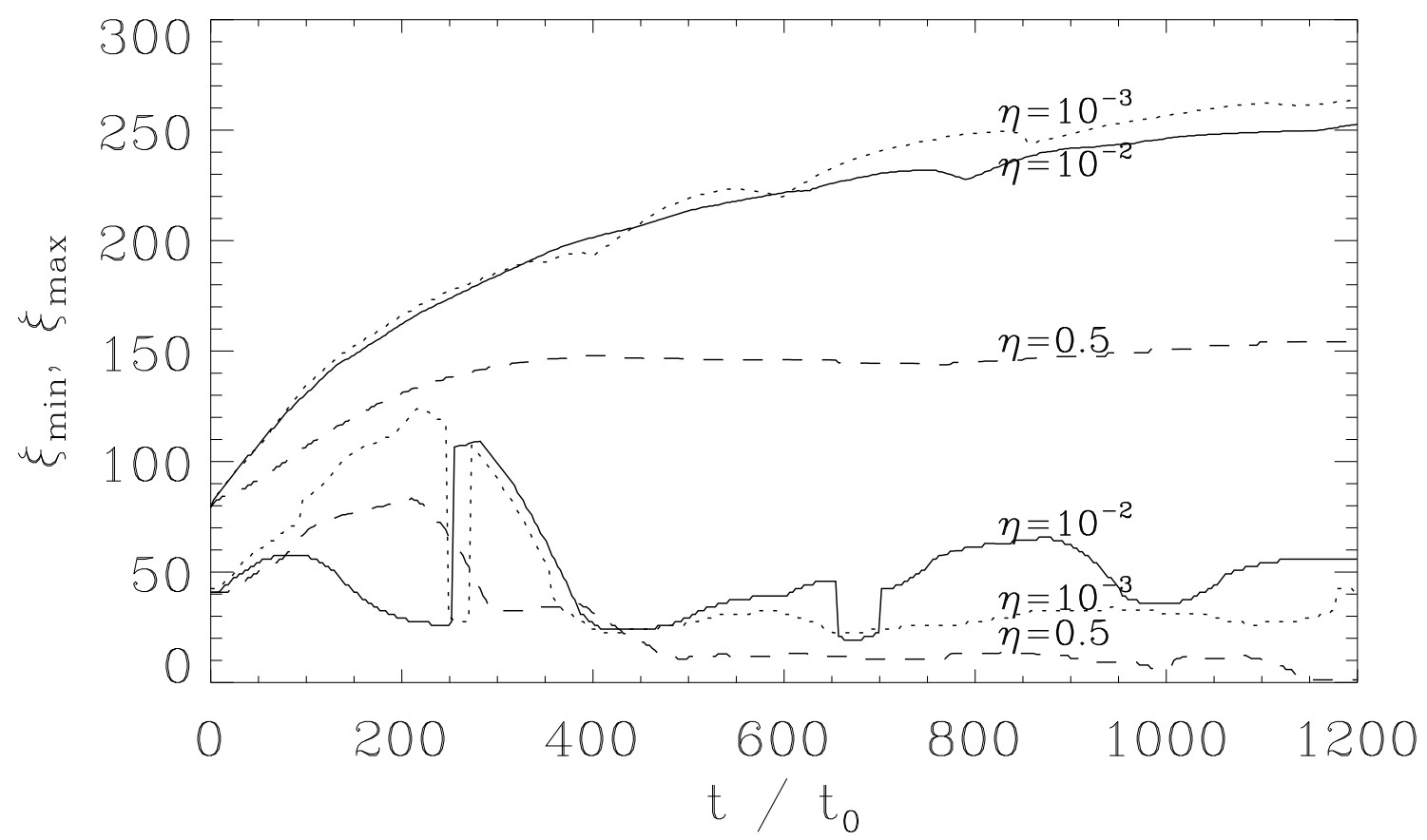

Fig. 8.- Maximum and minimum radial distances of bubble material from the galaxy nucleus, $\xi_{\max }$ and $\xi_{\min }$, as a functions of time for the cases with density contrasts $\eta=$ $0.5,10^{-2}, 10^{-3}$ (dashed, solid and dotted respectively). 


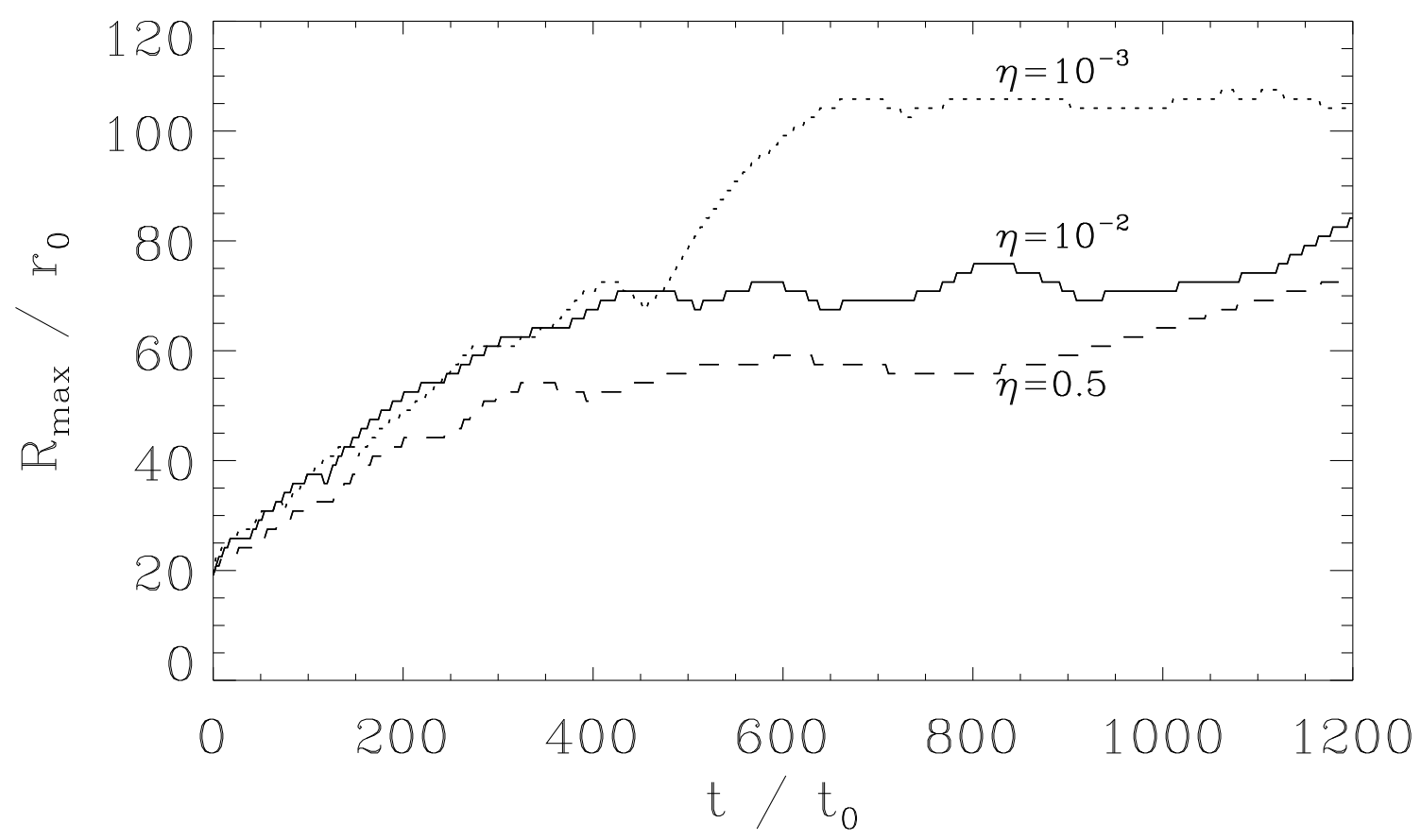

Fig. 9.- Extent of the bubble in the cylindrical radial direction $R_{\max }$ as a function of time for the cases with density contrasts $\eta=0.5,10^{-2}, 10^{-3}$. 


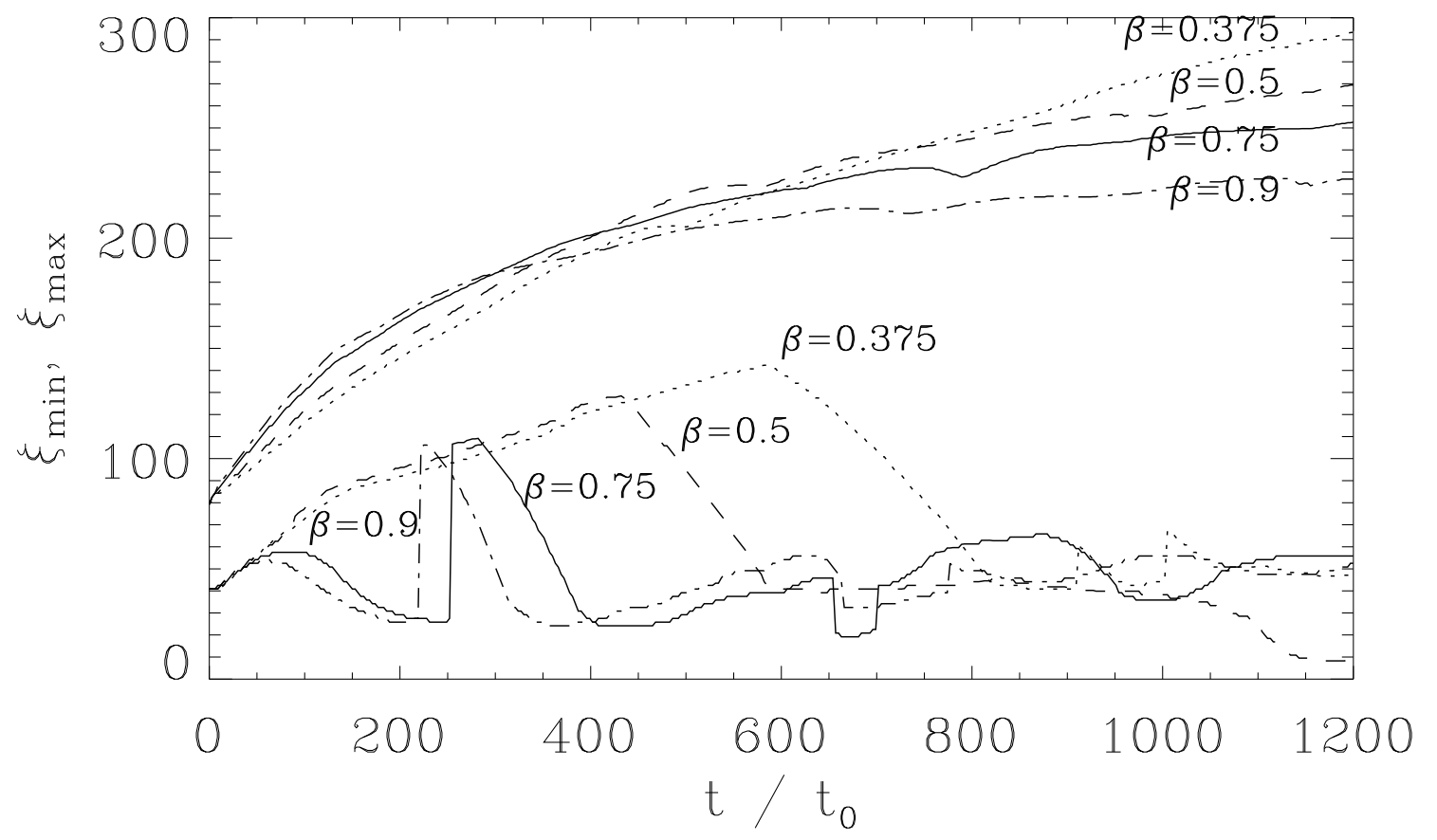

Fig. 10.- Maximum and minimum radial distances of bubble material from the galaxy nucleus, $\xi_{\max }$ and $\xi_{\min }$, as a functions of time for the cases with density contrast $\eta=10^{-2}$ and $\beta=0.375,0.5,0.75,0.9$ (dotted, dashed, solid and dot-dashed lines respectively). 

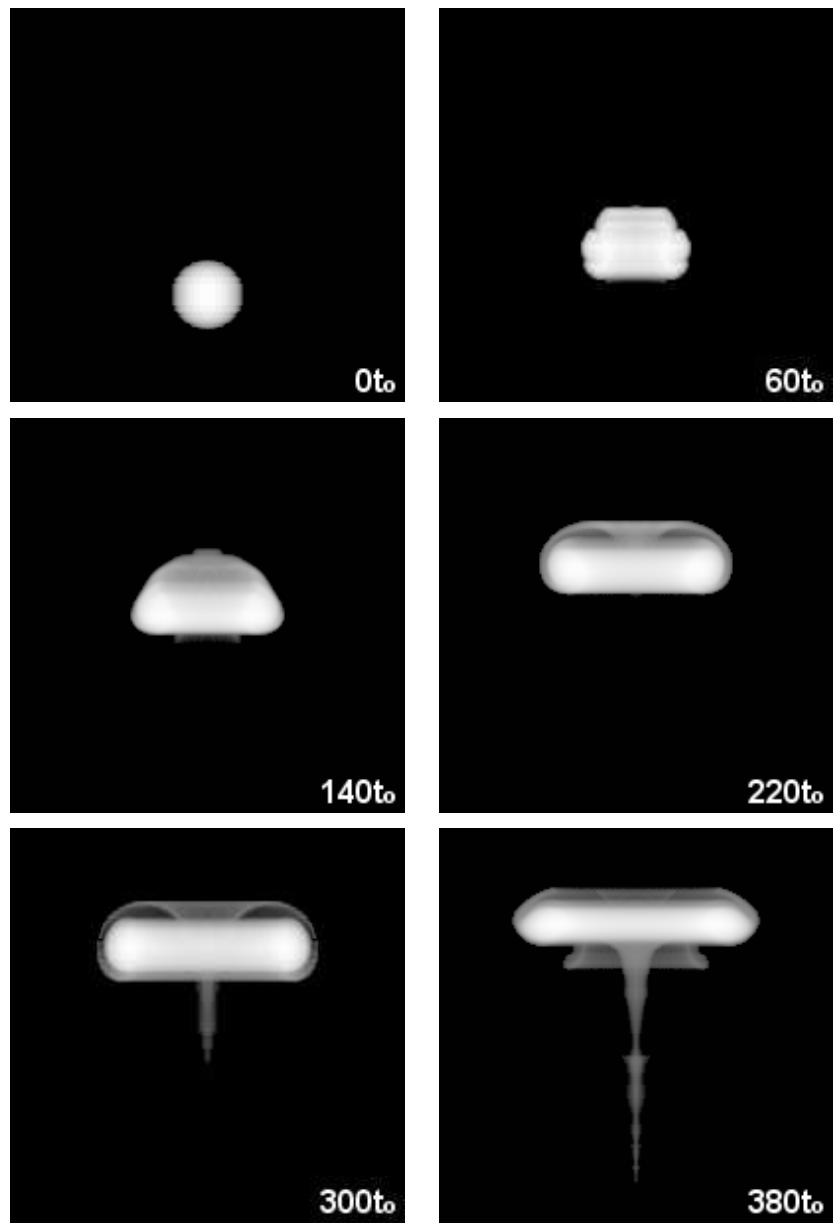

Fig. 11. - Raytraced simulated emission maps at different times. The $z$ axis is vertical at right angles to the line of sight. The origin is at the middle of the lower edge of each frame. This is the case of $\eta=10^{-3}$ and $\beta=0.75$. For the sake of clarity, results were obtained at triple the spatial resolution of our standard simulations. The frame at $t=140 t_{0}$ is in the "young torus" phase with wispy emission above the main bubble torus. We identify this stage with the observed radio morphology of the NML. 


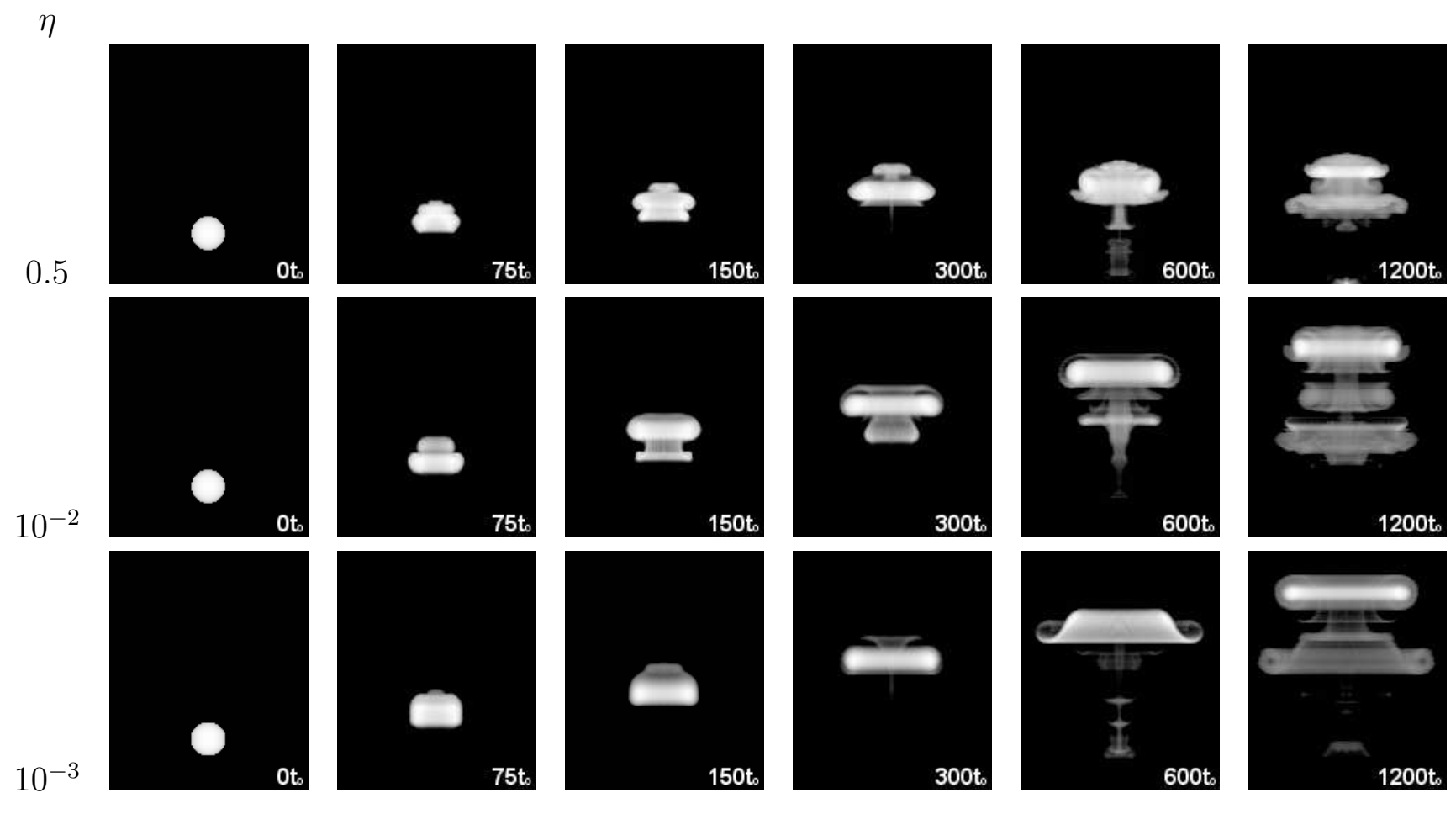

Fig. 12.- Raytraced simulated emission maps at different times, comparing the morphologies of different cases of $\eta$, with $\beta=0.75$. The axes are the same as in Figure 11. From top to bottom, the rows represent the cases $\eta=0.5,10^{-2}, 10^{-3}$ respectively. 

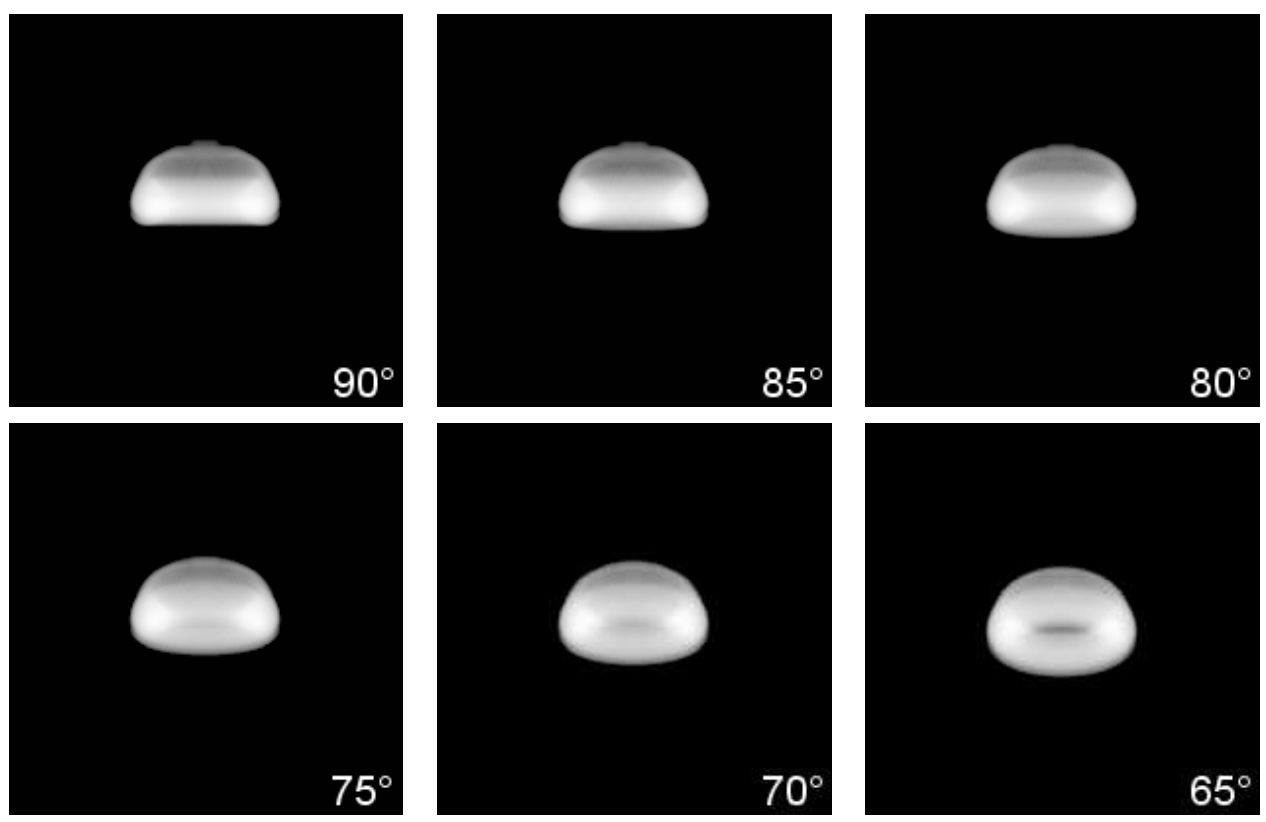

Fig. 13.- Raytraced simulated emission maps at different inclinations for the same case as Figure 11, i.e. $\eta=10^{-3}$ and $\beta=0.75$, at time $t=150 t_{0}$. 